\title{
Etude Ethnobotanique des plantes médicinales vendues dans le marché d'Abomey-Calavi au Bénin
}

\author{
A.C. ADOMOU ${ }^{1,2}$, H. YEDOMONHAN ${ }^{1 *}$, B. DJOSSA ${ }^{2}$, S. I. LEGBA ${ }^{2}$, M. OUMOROU ${ }^{2}$ et \\ A. AKOEGNINOU ${ }^{1}$ \\ ${ }^{I}$ Département de Biologie Végétale, Faculté des Sciences et Techniques, \\ Université d'Abomey-Calavi, 01 BP 4521 Cotonou, Bénin. \\ ${ }^{2}$ Laboratoire d'Ecologie Appliquée, Université d'Abomey-Calavi, BP 526 Cotonou. \\ *Auteur correspondant, E-mail :h.yedo@yahoo.fr
}

\section{RESUME}

La commercialisation et l'utilisation des essences médicinales constituent des pratiques très courantes en Afrique et en particulier au Bénin. Une enquête a été effectuée chez une seule vendeuse disposant de trois différents étalages. L'inventaire minutieux et détaillé des trois étals a duré 30 jours. Un total de 205 espèces végétales groupées en 181 genres et 74 familles ont été recensées. Les familles les plus représentées en termes de richesse spécifique sont: Leguminosae (15\%), Rubiaceae $(7 \%)$ et Euphorbiaceae $(7 \%)$. Ces espèces sont utilisées dans la formulation de 41 recettes pour traiter 37 maladies et symptômes. Le spectre biologique montre une nette prédominance des phanérophytes (68\%). L'analyse biogéographique montre une dominance des espèces guinéo-congolaises (29\%), pantropicales (29\%) et afrotropicales (10\%). Les tiges feuillées (67\%) et racines $(15 \%)$ constituent les organes les plus prisés. La décoction $(86 \%)$ et le savon $(12 \%)$ sont les formes pharmaceutiques fréquemment enregistrées. Les maladies et symptômes les plus fréquemment cités par les acheteurs sont : paludisme, diarrhée, stérilité, troubles menstruels, ictère, infections. Au nombre des plantes les plus vendues et rares, on peut citer: Khaya senegalensis, Monodora myristica, Xylopia aethiopica, Tetrapleura tetraptera, Acridocarpus smeathmannii et Entada gigas. La vente des plantes médicinales constitue non seulement une activité génératrice de revenu, mais contribue aussi aux soins de santé primaire, à la vulgarisation et la pérennisation du savoir endogène associé à l'utilisation des plantes médicinales.

(C) 2012 International Formulae Group. All rights reserved.

Mots clés : Plantes médicinales commercialisées, diversité, utilisations, rareté, Bénin.

\section{INTRODUCTION}

Après avoir combattu la médecine traditionnelle pendant longtemps, médecins et organismes de santé reconnaissent désormais la valeur et l'efficacité des traitements par les plantes. Ces dernières entrent également dans la composition de médicaments «modernes». Environ $80 \%$ de la population mondiale et plus de $90 \%$ de la population des pays en voie de développement y recourent pour les soins de santé primaire (Cunningham, 1993 ; Olsen, 2005 ; Pei, 2001 ; Jiofack et al., 2010). En effet, les substances élaborées au laboratoire se révèlent plus chères que les produits à base de plantes médicinales (Pamplona, 1999). Par conséquent, il existe un danger de voir se perdre le savoir lié aux plantes médicinales, même si les jeunes générations commencent à 
s'y intéresser. Ce type de savoir possède une réelle valeur culturelle et peut à terme permettre le développement de nouveaux médicaments pharmaceutiques.

Les marchés locaux de plantes médicinales concentrent, maintiennent et vulgarisent les connaissances empiriques sur l'utilisation des ressources phytogénétiques. De ce fait, ils contribuent à la conservation des plantes et du savoir endogène (Albuquerque et al., 2007).

L'exploitation durable des plantes médicinales commercialisées pourrait contribuer, non seulement à la préservation d'une part importante de la diversité biologique des forêts tropicales, mais également à l'amélioration des conditions de vie des communautés locales, grâce à la création de revenus et au traitement des maladies. Le secteur du commerce local des plantes médicinales au Bénin suscite de plus en plus d'intérêt, tant pour sa contribution au bien-être (revenus, soins de santé) de la population que pour son potentiel commercial dans le développement de nouveaux produits médicaux. En reconnaissant donc l'importance des plantes médicinales et en mettant à profit les connaissances des guérisseurs traditionnels, des phytothérapeutes, des vendeurs et des collecteurs, il y a une occasion exceptionnelle de relier trois des principaux indicateurs de la pauvreté : l'environnement, le développement rural et la santé. Avant de pouvoir réaliser ce développement, des informations essentielles de base (diversité, demande, rareté, lieux de collecte etc.) sont requises pour garantir la survie des acteurs, l'exploitation durable des ressources et la conservation de la biodiversité végétale. Les travaux relatifs à l'inventaire des plantes médicinales vendues dans les marchés locaux sont rares en Afrique subsaharienne (Maiga et al., 2005).

L'inventaire de marché des plantes médicinales n'est pas souvent une tâche aisée. L'une des difficultés majeures rencontrées est la réticence des vendeurs lors des enquêtes ethnobotaniques (Gil et al., 2003). Ce qui affecte inévitablement la qualité des données comme la richesse botanique des étals de marché et la diversité des usages. Généralement, les ethnobotanistes se préoccupent plus du nombre de vendeurs et d'étals à inventorier que du temps nécessaire pour se familiariser avec les informateurs afin de rendre l'inventaire exhaustif. Ainsi, un inventaire approfondi et minutieux des étals de plantes médicinales au marché pourrait conduire à des valeurs plus élevées de diversité taxonomique. Les objectifs de la présente étude sont de :1) inventorier les étals de plantes médicinales et les organes végétaux commercialisés auprès d'une vendeuse suite à son accord, 2) recenser leurs usages et les maladies traitées, 3) recueillir la perception de la vendeuse et des collecteurs sur la rareté des plantes médicinales, 4) déterminer les caractéristiques biologiques et biogéographiques des espèces.

\section{MATERIEL ET METHODES \\ Site d'étude}

L'étude a été conduite au sud du Bénin (Afrique de l'Ouest) dans le marché d'Abomey-Calavi (latitude, 06 $27^{\circ} 0^{\prime}$ ' $N$; longitude, $02^{\circ} 21^{\prime} 0$ ' 'E; altitude, $12 \mathrm{~m}$ ). Le climat est de type subéquatorial avec deux saisons pluvieuses et deux saisons sèches. Le total pluviométrique annuel est de $1300 \mathrm{~mm}$ d'eau. L'hygrométrie reste élevée toute l'année avec une moyenne de $80 \%$. La température reste élevée $\left(26,6{ }^{\circ} \mathrm{C}\right)$ avec une amplitude thermique pouvant atteindre $7{ }^{\circ} \mathrm{C}$ (Akoègninou, 2004). La ville s'étend sur 650 $\mathrm{km}^{2}(0,48 \%$ de la superficie nationale $)$ et compte 307745 habitants avec une densité de 473,5 habitants par $\mathrm{km}^{2}$ (Anonyme, 2002). L'ethnie dominante dans la commune d'Abomey-Calavi est aïzo (84\%) suivie des fon, yoruba et adja. Les principales activités sont l'agriculture, la pêche, l'élevage et le commerce.

\section{Enquêtes ethnobotaniques \\ Une vendeuse de plantes médicinales, expérimentée dans la pratique de la}


phytothérapie, a été sélectionnée. Elle exerce ce commerce depuis plus d'une décennie et donc est susceptible de fournir des informations utiles et originales sur l'usage populaire des plantes médicinales. Sur la base d'un accord établi, elle a accepté coopérer dans l'inventaire détaillé de ses trois étals de plantes médicinales. Une étudiante du niveau Licence du Département de Génie de l'Environnement de l'Ecole Polytechnique d'Abomey-Calavi (EPAC) de l'Université d'Abomey-Calavi a été mise à contribution pour ce travail et a été accueillie par la vendeuse comme stagiaire. Ceci a permis de faire un inventaire détaillé des espèces de plantes médicinales vendues. L'interview est basée sur le dialogue en langue locale (aïzo ou fon) accompagné de l'achat d'échantillons de plantes médicinales qui sont mis en herbier. Les rubriques de l'interview concernent notamment des informations sur les parties de la plante utilisée, les méthodes de préparation, les maladies guéries et la posologie. La collecte des données ethnobotaniques a duré 30 jours au cours desquels le flux des acheteurs et la fréquence de vente des plantes médicinales sont appréciés et les diverses maladies traitées par la vendeuse phytothérapeute sont recensées. Les enquêtes basées sur des interrogations directes ou des observations ont aussi porté sur les localités de collecte des organes végétaux et la rareté des plantes. En plus de la vendeuse phytothérapeute, 10 collecteurs de plantes et 50 acheteurs de plantes ont été interviewés de façon aléatoire. Les échantillons des plantes collectés ont été identifiés à l'Herbier National du Bénin. La Nomenclature utilisée est celle de la Flore Analytique du Bénin (Akoègninou et al., 2006).

Les informations sur le « diagnostic des maladies » (symptômes ou effets physiologiques) ont été recueillies auprès des médecins, infirmiers ou agents de santé locaux et complétées par la revue bibliographique (Adjanohoun et al., 1989). Pour une exploitation pratique des données et une harmonisation avec le système international, les problèmes de santé cités ont été distingués en grands groupes de maladies selon la dernière classification des maladies proposée par l'Organisation Mondiale de la Santé et adaptée par l'Organisation de l'Unité Africaine (OUA) pour la pharmacopée africaine (Adjanohoun et al., 1996). Les données collectées ont été enregistrées sur le tableur Excel puis analysées.

Pour apprécier l'importance de la demande des espèces par les acheteurs, cinq classes de fréquence ont été définies : I : Très faiblement vendues (inférieur à 1 personne par jour ; II :Faiblement vendues (1 à 5 personnes par jour); III : Assez vendu (5- 9 personnes par jour); IV : Fréquemment vendu (10-20 personnes par jour); V: Très fréquemment vendu (supérieur à 20 personnes par jour).

Les types biologiques des espèces ont été déterminés en suivant le système de Raunkiaer (1934) adapté à la végétation tropicale (Aké Assi, 2001; 2002). La caractérisation biogéographique des espèces a été faite en utilisant les types de distribution de White (1984).

\section{RESULTATS \\ Diversité des espèces}

Au total, 205 espèces utilisées comme plantes médicinales ont été identifiées et inventoriées sur les trois étals de la vendeuse phytothérapeute (annexe 1). Elles sont réparties dans 181 genres et 74 familles. Parmi elles, environ 22\% (soit 46 espèces) sont cultivées ou plantées. Au nombre de celles-ci, on peut citer: Cyperus esculentus, Caesalpinia bonduc, Catharanthus roseus, Cocos nucifera, Mangifera indica, Casuarina equisetifolia, Newbouldia laevis, Croton zambesicus, Commiphora africana, Cola acuminata, Piper guineense et Ocimum spp. Celles comme Argemone mexicana, Commiphora africana, Heliotropium indicum, Kalankoe crenata, Chenopodium ambrosioides.

Les familles ayant les richesses spécifiques les plus élevées sont: Leguminosae (31 espèces, soit $15 \%)$, 
Rubiaceae (15 espèces, soit 7\%), Euphorbiaceae (9 espèces, soit $4 \%$ ) et Apocynaceae (7 espèces, soit $3 \%$ ).

\section{Spectres biologique et phytogéographique}

Le spectre biologique des espèces recensées montre que les phanérophytes sont largement dominants avec un taux de $68 \%$. Elles sont suivies des thérophytes (17\%), des chaméphytes (7\%), des géophytes $(6 \%)$ et autres (2\%). Les lianes représentent $15 \%$ de l'ensemble des espèces recensées (annexe 1).

Le spectre biogéographique des espèces recensées montre une dominance des espèces guinéo-congolaises (29\%), pantropicales $(29 \%)$, afro-tropicales $(10 \%)$, soudano-guinéennes (9\%) et soudaniennes (9\%).

\section{Parties de la plante, formes pharmaceutiques et maladies traitées}

La Figure 1 présente les pourcentages des organes végétaux recensés sur l'étal. Les tiges feuillées sont les plus représentées (67\%), suivies des racines (15\%), écorces $(7 \%)$ et fruits $(5 \%)$.

Les 205 espèces recensées interviennent dans 41 recettes. La décoction $(86 \%)$ et le savon (12\%) sont les formes pharmaceutiques les plus citées; la macération (aqueuse ou alcoolique) est rarement mentionnée $(0,5 \%)$. Les recettes indiquées par la vendeuse phytothérapeute sont utilisées pour traiter 37 maladies et symptômes dont les plus fréquemment citées sont: paludisme, diarrhées, stérilité féminine, troubles menstruels, ictère, accouchements difficiles et infections (Figure 2).

Certains organes sont qualifiés d'ingrédients auxiliaires par la vendeuse et représentent $13 \%$ des espèces inventoriées. Selon elle, les ingrédients auxiliaires sont des organes végétaux généralement aromatiques qui accompagnent la préparation des recettes ; ils permettent généralement de traiter les infections opportunistes comme dans le cas du paludisme. Il s'agit des fruits de: Xylopia aethiopica, Tetrapleura tetraptera, Schrebera arborea, Acacia nilotica, Kigelia africana, Adenopus breviflorus; des graines de: Caesalpinia bonduc, Garcinia cola, Monodora myristica, Entada gigas; des bulbes de: Allium cepa, Pancratium tenuifolium; des rhizomes de: Cyperus esculentus, Curculigo pilosa et des tubercules de Anchomanes difformis.

\section{Espèces les plus vendues}

Le Tableau 1 présente les espèces végétales dont les organes sont les plus demandées; ce qui fait que la pression anthropique sur ces espèces est très forte. Les espèces telles que Khaya senegalensis, Monodora myristica, Xylopia aethiopica, Tetrapleura tetraptera, Acridocarpus smeathmannii, Cleistopholis patens, Entada gigas, Kigelia africana, Pavetta crassipes, Remirea maritima se font de plus en plus rares selon les enquêtés. Ces espèces interviennent dans le traitement des maladies courantes telles que les infections (13 espèces) et le paludisme (3 espèces). Les espèces telles que Catharanthus roseus, Lagenaria siceraria, Ocimum canum, Argemone mexicana et Cola acuminata sont plantées. Les fleurs séchées de Eugenia aromatica sont importées du Nigeria.

$\mathrm{Au}$ nombre des espèces les plus sollicitées, certaines comme Cucumis metuliferus, Lagenaria siceraria, Kigelia africana, Securidaca longepedunculata et Acridocarpus smeathmannii interviennent dans des formulations réalisées par la vendeuse. Celles-ci sont particulièrement très demandées par la population pour le traitement d'affections spécifiques telles que les kystes, sinusites, infections intestinales infantiles, etc.

Les espèces très rares selon la perception des collecteurs et de la vendeuse sont indiquées dans le Tableau 1. La notion de rareté selon eux, serait liée à la difficulté de trouver la plante qui peut être fonction de la distance parcourue. Les espèces telles que Tylophora cameroonica et Argemone mexicana sont plantées dans les maisons et sont difficiles à trouver; Argemone mexicana 
est une plante annuelle et n'est disponible que pendant la saison pluvieuse. Certaines espèces telles que Caesalpinia bonduc, Acridocarpus smeathmannii, Carissa spinarum, Khaya senegalensis, Plumbago zeylanica sont difficiles à trouver et correspondent généralement à celles qui sont vendues chères sur les marchés. Le prix de la botte varie entre 300 et 500 FCFA (ou 0,5-1 dollar US) pour ces espèces. Cependant, le prix des organes végétaux vendus est fonction aussi de l'efficacité de la plante. Les collecteurs de plantes ont indiqué que le prélèvement des racines de Caesalpinia bonduc est préjudiciable pour la plante; l'efficacité de ses racines dans le traitement des faiblesses sexuelles, infections et prostatites est aussi reconnue par les acheteurs. C'est aussi le cas des racines de Gardenia ternifolia dont l'efficacité contre l'hypertension est constamment indiquée par la vendeuse phytothérapeute et les acheteurs.

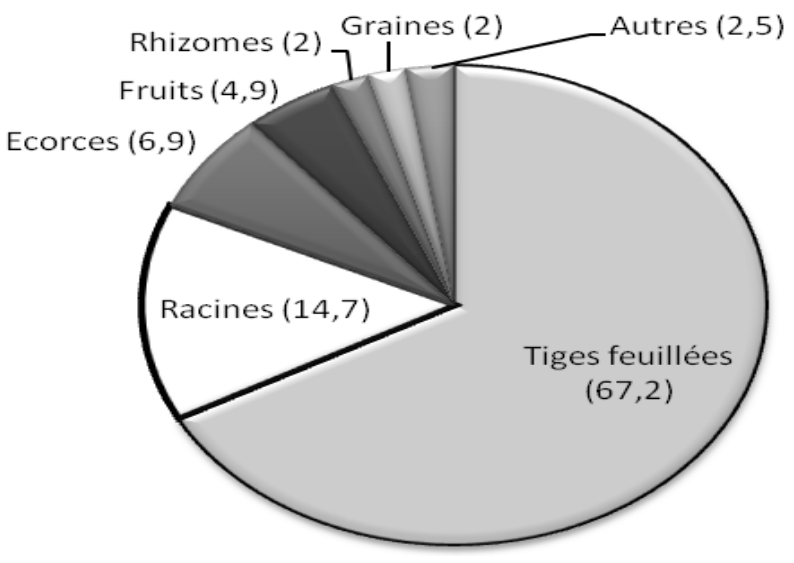

Figure 1 : Fréquence des organes végétaux sur l'étal de marché.

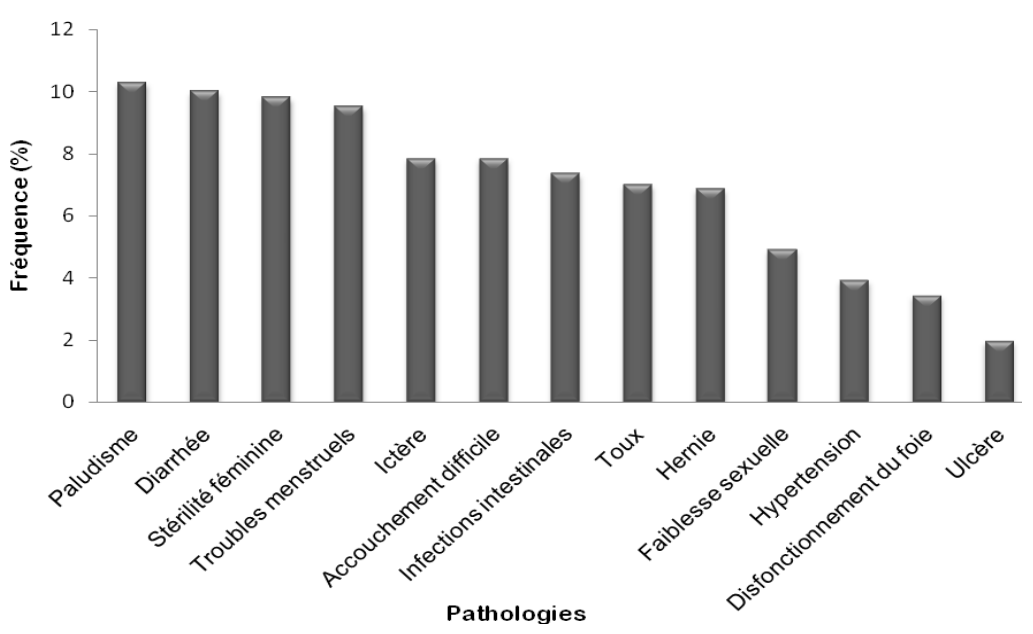

Figure 2 : Maladies fréquemment traitées par la vendeuse phytothérapeute. 
A.C. ADOMOU et al. / Int. J. Biol. Chem. Sci. 6(2): 745-772, 2012

Tableau 1: Espèces les plus vendues et rares selon la perception des collecteurs et de la vendeuse.

\begin{tabular}{|c|c|c|c|c|}
\hline Espèces & Famille & Organes & $\begin{array}{c}\text { Pathologies traitées (ou } \\
\text { autres usages) selon la } \\
\text { vendeuse } \\
\end{array}$ & $\begin{array}{l}\text { Fréquence } \\
\text { de vente }\end{array}$ \\
\hline $\begin{array}{l}\text { Acridocarpus } \\
\text { smeathmannii( }(\mathbf{R})\end{array}$ & Malpighiaceae & Racine & Asthénie sexuelle, anémie & IV \\
\hline Argemone mexicana $(\mathbf{R})$ & Papaveraceae & Tige feuillée & $\begin{array}{l}\text { Ictère, facilitation chute du } \\
\text { cordon ombilical et } \\
\text { prévention des maux de } \\
\text { ventre chez le nouveau-né }\end{array}$ & III \\
\hline Catharanthus roseus & Apocynaceae & Racine & Hypertension & $\mathrm{V}$ \\
\hline Cissus populnea & Vitaceae & Racine & $\begin{array}{c}\text { Asthénie sexuelle, stérilité } \\
\text { (masculine et féminine) }\end{array}$ & IV \\
\hline Cleistopholis patens & Annonaceae & Ecorce & $\begin{array}{l}\text { Kyste, fibromes, myomes et } \\
\text { stérilité féminine }\end{array}$ & IV \\
\hline Cola acuminata & Malvaceae & Graine, racine & $\begin{array}{l}\text { Rougeole (infections), } \\
\text { hypertension }\end{array}$ & III \\
\hline Cucumis metuliferus & Cucurbitaceae & Fruit & $\begin{array}{l}\text { Kyste, fibromes, myomes } \\
\text { (chez les femmes) }\end{array}$ & V \\
\hline Cucurligo pilosa & Hypoxidaceae & Tubercule & Infections & $\mathrm{V}$ \\
\hline Desmodium ramossissimum & $\begin{array}{l}\text { Leguminosae- } \\
\text { Papilionoideae }\end{array}$ & Tige feuillée & Accouchement difficile & III \\
\hline Entada gigas & $\begin{array}{l}\text { Leguminosae- } \\
\text { Mimosoideae }\end{array}$ & Graine & Infections & V \\
\hline Eugenia aromatica & Myrtaceae & fleurs & Infections & $\mathrm{V}$ \\
\hline Kedrostis foetidissima $(\mathbf{R})$ & Cucurbitaceae & Tige feuillée & Magique (porte chance) & IV \\
\hline Khaya senegalensis(R) & Meliaceae & Ecorce & Infections, maux de ventre & IV \\
\hline
\end{tabular}


A.C. ADOMOU et al. / Int. J. Biol. Chem. Sci. 6(2): 745-772, 2012

\begin{tabular}{|c|c|c|c|c|}
\hline Kigelia africana & Bignoniaceae & Fruit et écorce & Infections & $\mathrm{V}$ \\
\hline Lagenaria siceraria & Cucurbitaceae & Fruit & Infections & $\mathrm{V}$ \\
\hline Lippia multiflora & Verbenaceae & Tige feuillée & $\begin{array}{l}\text { Infections dermatologiques, } \\
\text { hypertension }\end{array}$ & V \\
\hline Monodora myristica & Annonaceae & Graine & Infections & $\mathrm{V}$ \\
\hline Ocimum canum & Lamiaceae & Tige feuillée & Infections & IV \\
\hline Pancratium tenuifolium(R) & Amaryllidaceae & Bulbe & Toux & III \\
\hline Pavetta corymbosa(R) & Rubiaceae & Tige feuillée & Paludisme & $\mathrm{V}$ \\
\hline Pavetta crassipes & Rubiaceae & Tige feuillée & Paludisme & $\mathrm{V}$ \\
\hline $\begin{array}{l}\text { Psorospermum } \\
\text { glaberrimum }(\mathbf{R})\end{array}$ & Clusiaceae & Tige feuillée & Infections dermatologiques & III \\
\hline Remirea maritima & Cyperaceae & Feuille & Troubles de dentition & IV \\
\hline Securidaca longepedunculata & Polygalaceae & Racines & Sinusite, toux & IV \\
\hline Senna siamea & $\begin{array}{l}\text { Leguminosae- } \\
\text { Caesalpinioideae }\end{array}$ & $\begin{array}{l}\text { Tige feuillée, } \\
\text { Racine, Ecorce }\end{array}$ & $\begin{array}{l}\text { Paludisme, hypertension, } \\
\text { fièvre typhoïde (infection) }\end{array}$ & V \\
\hline Tamarindus indica $\mathbf{( R )}$ & $\begin{array}{c}\text { Leguminosae- } \\
\text { Caesalpinioideae }\end{array}$ & Ecorce & Paludisme & III \\
\hline Terminalia glaucescens $(\mathbf{R})$ & Combretaceae & Racine & Toux & III \\
\hline Tetrapleura tetraptera & $\begin{array}{l}\text { Leguminosae- } \\
\text { Mimosoideae }\end{array}$ & Fruit & Infections & V \\
\hline Trilepisium madagacariensis & Moraceae & Ecorce & Troubles menstruels & IV \\
\hline Tylophora cameroonica(R) & Asclepiadaceae & Feuilles & Magique (protection) & III \\
\hline Xylopia aethiopica & Annonaceae & Fruit & Infections & $\mathrm{V}$ \\
\hline
\end{tabular}

I : Très faiblement vendues (inférieur à 1 personne par jour ) II :Faiblement vendues (1 à 5 personnes par jour) ; III : Assez vendu (5- 9 personnes par jour) ; IV : Fréquemment vendues (10-20 personnes par jour) : V : Très fréquemment vendues (supérieur à 20 personnes par jour).

Les espèces très rares (selon la perception des collecteurs et de la vendeuse) sont accompagnées du symbole $(\mathbf{R})$. 
A.C. ADOMOU et al. / Int. J. Biol. Chem. Sci. 6(2): 745-772, 2012

Annexe 1: Liste des espèces inventoriées sur les étals de plantes médicinales de la vendeuse.

\begin{tabular}{|c|c|c|c|c|c|c|c|}
\hline $\begin{array}{l}\text { Espèces de } \\
\text { plantes }\end{array}$ & $\begin{array}{c}\text { Noms vernaculaires } \\
\text { (langue Fon) }\end{array}$ & Familles & TB & $\mathbf{T P}$ & Org & Maladies/Symptômes & Fph et Mad \\
\hline $\begin{array}{l}\text { Amaranthus } \\
\text { viridis }\end{array}$ & Amandjin & Amaranthaceae & Th & $\mathrm{Pt}$ & Tfe & $\begin{array}{c}\text { Bronchite, magique } \\
\text { (protection) }\end{array}$ & Savon (bain) \\
\hline Acacia nilotica & Vanli & $\begin{array}{l}\text { Leguminosae- } \\
\text { Mimosoideae }\end{array}$ & $\mathrm{mph}$ & $S$ & Fr & Infections & $\begin{array}{l}\text { Décoction } \\
\text { (or) }\end{array}$ \\
\hline Acalypha crenata & Faux Djividjivi & Euphorbiaceae & Th & At & Tfe & Stérilité & $\begin{array}{l}\text { Décoction } \\
\text { (or) }\end{array}$ \\
\hline $\begin{array}{l}\text { Acanthospermum } \\
\text { hispidum }\end{array}$ & Ahanglon & Asteraceae & Th & $\mathrm{Pt}$ & Tfe & Ictère, paludisme & $\begin{array}{l}\text { Décoction } \\
\text { (or) }\end{array}$ \\
\hline $\begin{array}{l}\text { Acanthus } \\
\text { montanus }\end{array}$ & Dèbio & Acanthaceae & $\mathrm{Ch}$ & $\mathrm{GC}$ & Tfe & $\begin{array}{l}\text { Magique (porte } \\
\text { chance) }\end{array}$ & Savon (bain) \\
\hline $\begin{array}{l}\text { Acmella } \\
\text { uliginosa }\end{array}$ & Wlélékpékpé & Asteraceae & Th & $\mathrm{Pt}$ & Tfe & $\begin{array}{l}\text { Magique (porte } \\
\text { chance) }\end{array}$ & Savon (bain) \\
\hline $\begin{array}{l}\text { Acridocarpus } \\
\text { smeathmannii }\end{array}$ & Gbanguinado & Malpighiaceae & Lmph & GC & $\mathrm{Ra}$ & $\begin{array}{c}\text { Faiblesse sexuelle } \\
\text { (homme), asthénie, } \\
\text { anémie }\end{array}$ & $\begin{array}{l}\text { Décoction } \\
\text { (or) }\end{array}$ \\
\hline $\begin{array}{l}\text { Adansonia } \\
\text { digitata }\end{array}$ & Kpassagoto & Bombacaceae & $\mathrm{mPh}$ & $\mathrm{SZ}$ & $\mathrm{Ec}$ & $\begin{array}{l}\text { Kyste, fibrome, } \\
\text { myome, toux }\end{array}$ & $\begin{array}{l}\text { Décoction } \\
\text { (or) }\end{array}$ \\
\hline $\begin{array}{l}\text { Adenopus } \\
\text { breviflorus }\end{array}$ & Weblikpo & Cucurbitaceae & Lnph & At & Fr & Infections & $\begin{array}{l}\text { Décoction } \\
\text { (or) }\end{array}$ \\
\hline Aerva lanata & Akoèman & Amaranthaceae & Th & $\mathrm{Pt}$ & Tfe & $\begin{array}{l}\text { Magique (porte } \\
\text { chance) }\end{array}$ & $\begin{array}{l}\text { Décoction } \\
\text { (or) }\end{array}$ \\
\hline *Aframomum & Ataku & Zingiberaceae & $\mathrm{Gr}$ & GC & $\mathrm{Fr}$ & Maux de ventre & Décoction \\
\hline
\end{tabular}


A.C. ADOMOU et al. / Int. J. Biol. Chem. Sci. 6(2): 745-772, 2012

\begin{tabular}{|c|c|c|c|c|c|c|c|}
\hline melegueta & & & & & & & (or) \\
\hline $\begin{array}{l}\text { Aframomum } \\
\text { sceptrum }\end{array}$ & Atabougoudou & Zingiberaceae & $\mathrm{Gr}$ & GC & Tfe & $\begin{array}{l}\text { Magique (porte } \\
\text { chance) }\end{array}$ & Savon (bain) \\
\hline $\begin{array}{l}\text { Agelaea } \\
\text { pentagyna }\end{array}$ & Ahanhlazu & Connaraceae & Lnph & $\mathrm{GC}$ & Tfe & $\begin{array}{c}\text { Retard staturo- } \\
\text { pondéral (enfants) }\end{array}$ & $\begin{array}{l}\text { Décoction } \\
\text { (or) }\end{array}$ \\
\hline *Allium cepa & Agnoman & Alliaceae & $\mathrm{Gb}$ & $\mathrm{Pt}$ & $\mathrm{Bu}$ & Infections & $\begin{array}{l}\text { Décoction } \\
\text { (or) }\end{array}$ \\
\hline $\begin{array}{l}\text { Allophyllus } \\
\text { africanus }\end{array}$ & Yanbobo & Sapindaceae & $\mathrm{mph}$ & $\mathrm{Pt}$ & Tfe & Evanouissement & $\begin{array}{l}\text { Décoction } \\
\text { (or) }\end{array}$ \\
\hline * Aloe spp & Aloès & Asphodelaceae & $\mathrm{Gb}$ & $?$ & $\mathrm{Fe}$ & Infections, Plaies & $\begin{array}{c}\text { Jus } \\
\text { (application } \\
\text { locale) }\end{array}$ \\
\hline $\begin{array}{l}\text { Ampelocissus } \\
\text { bombycina }\end{array}$ & Tèkplè & Vitaceae & Lmph & S & Tfe & Paludisme & $\begin{array}{l}\text { Décoction } \\
\text { (or) }\end{array}$ \\
\hline $\begin{array}{l}\text { Anchomanes } \\
\text { difformis }\end{array}$ & Godoe & Araceae & Gt & GC & $\mathrm{Tu}$ & Infections & $\begin{array}{l}\text { Décoction } \\
\text { (or) }\end{array}$ \\
\hline $\begin{array}{l}\text { Annona } \\
\text { sengalensis }\end{array}$ & Wiglédo & Annonaceae & nph & $\mathrm{SZ}$ & $\mathrm{Ra}$ & $\begin{array}{c}\text { Hernie, maux de } \\
\text { ventre }\end{array}$ & $\begin{array}{l}\text { Décoction } \\
\text { (or) }\end{array}$ \\
\hline $\begin{array}{l}\text { Anogeissus } \\
\text { leiocarpa }\end{array}$ & Hlihon & Combretaceae & $\mathrm{mPh}$ & $\mathrm{S}$ & Tfe & Infections & $\begin{array}{l}\text { Décoction } \\
\text { (or) }\end{array}$ \\
\hline $\begin{array}{l}* \text { Argemone } \\
\text { mexicana }\end{array}$ & Houètchégnon & Papaveraceae & Th & $\mathrm{Pt}$ & Tfe & $\begin{array}{c}\text { Ictère, soins du } \\
\text { nombril (nouveau-né) }\end{array}$ & $\begin{array}{l}\text { Décoction } \\
\text { (or) }\end{array}$ \\
\hline Aspilia africana & Houéflougbéton & Asteraceae & Th & SG & Tfe & Infections & $\begin{array}{l}\text { Décoction } \\
\text { (or) }\end{array}$ \\
\hline $\begin{array}{l}\text { Biophytum } \\
\text { petersianum }\end{array}$ & Kikè & Oxalidaceae & Th & $\mathrm{Pt}$ & Tfe & $\begin{array}{l}\text { Magique (porte } \\
\text { chance) }\end{array}$ & Savon (bain) \\
\hline
\end{tabular}


A.C. ADOMOU et al. / Int. J. Biol. Chem. Sci. 6(2): 745-772, 2012

\begin{tabular}{|c|c|c|c|c|c|c|c|}
\hline * Blighia sapida & Lisè & Sapindaceae & $\mathrm{mPh}$ & $\mathrm{Pt}$ & Tfe & $\begin{array}{l}\text { Ulcère, fièvre } \\
\text { typhoïde }\end{array}$ & Savon (bain) \\
\hline Boerhavia erecta & Cachiyonawi & Nyctaginaceae & $\mathrm{Th}$ & $\mathrm{Pt}$ & Tfe & $\begin{array}{l}\text { Traite le kyste, } \\
\text { myome, fibrome }\end{array}$ & Savon (bain) \\
\hline $\begin{array}{l}\text { Borassus } \\
\text { aethiopum }\end{array}$ & Agontéglé & Arecaceae & $\mathrm{mPh}$ & $\mathrm{SZ}$ & FrH & $\begin{array}{l}\text { Traite les problèmes } \\
\text { de faiblesse sexuelle }\end{array}$ & $\begin{array}{c}\text { Alcoolature } \\
\text { (or) }\end{array}$ \\
\hline $\begin{array}{l}\text { Bridelia } \\
\text { ferruginea }\end{array}$ & Honssoukékédo & Euphorbiaceae & $\mathrm{mph}$ & SG & $\mathrm{Ra}$ & $\begin{array}{l}\text { Traite le Kyste, } \\
\text { fibrome,myome }\end{array}$ & $\begin{array}{l}\text { Décoction } \\
\text { (or) }\end{array}$ \\
\hline $\begin{array}{l}\text { * Caesalpinia } \\
\text { bonduc }\end{array}$ & Adjikoui & $\begin{array}{c}\text { Leg.- } \\
\text { Caesalpinioideae }\end{array}$ & Lnph & $\mathrm{Pt}$ & $\begin{array}{l}\text { Tfe- } \\
\text { Gr- } \\
\mathrm{Ra}\end{array}$ & $\begin{array}{c}\text { Diabète-faiblesse } \\
\text { sexuelle, prostatite, } \\
\text { infections }\end{array}$ & $\begin{array}{l}\text { Décoction } \\
\text { (or) }\end{array}$ \\
\hline $\begin{array}{l}\text { * Caesalpinia } \\
\text { pucherrima }\end{array}$ & Orgueil de Chine & $\begin{array}{c}\text { Leg.- } \\
\text { Caesalpinioideae }\end{array}$ & nph & $\mathrm{Pt}$ & Tfe & Infections & $\begin{array}{l}\text { Décoction } \\
\text { (or, bain) }\end{array}$ \\
\hline $\begin{array}{l}\text { Calyptrochilum } \\
\text { christyanum }\end{array}$ & Avaman & Orchidaceae & Ep & SG & Tfe & $\begin{array}{l}\text { Magique (porte } \\
\text { chance) }\end{array}$ & Savon (bain) \\
\hline $\begin{array}{l}\text { Cardiospermum } \\
\text { halicaccabum }\end{array}$ & Ahonman & Sapindaceae & Lmph & $\mathrm{Pt}$ & Tfe & $\begin{array}{l}\text { Fragilité de la tête } \\
\text { chez le nouveau-né }\end{array}$ & $\begin{array}{l}\text { Décoction } \\
\text { (or) }\end{array}$ \\
\hline Carissa spinarum & Ahanzodo & Apocynaceae & nph & $\mathrm{Pt}$ & Ec & $\begin{array}{c}\text { Faiblesse sexuelle, } \\
\text { maux de ventre }\end{array}$ & $\begin{array}{c}\text { Décoction } \\
\text { (or) }\end{array}$ \\
\hline Carpolobia lutea & Aviado & Polygalaceae & $\mathrm{mph}$ & $\mathrm{GC}$ & $\mathrm{Ra}$ & Faiblesse sexuelle & $\begin{array}{l}\text { Décoction } \\
\text { (or) }\end{array}$ \\
\hline Cassia sieberiana & Hinhami & $\begin{array}{c}\text { Leg.- } \\
\text { Caesalpinioideae }\end{array}$ & $\mathrm{mPh}$ & $S$ & Tfe & Infections & $\begin{array}{l}\text { Décoction } \\
\text { (or) }\end{array}$ \\
\hline $\begin{array}{l}\text { Cassytha } \\
\text { filiformis }\end{array}$ & Agbègbèkan & Lauraceae & Par & $\mathrm{Pt}$ & Tfe & $\begin{array}{c}\text { Accouchement } \\
\text { difficile }\end{array}$ & $\begin{array}{c}\text { Décoction } \\
\text { (or) }\end{array}$ \\
\hline $\begin{array}{l}\text { * Casuarina } \\
\text { equisetifolia }\end{array}$ & Filaogoto & Casuarinaceae & $\mathrm{MPh}$ & At & $\mathrm{Ec}$ & $\begin{array}{c}\text { Kyste, myome, } \\
\text { fibrome et infections }\end{array}$ & $\begin{array}{l}\text { Décoction } \\
\text { (or) }\end{array}$ \\
\hline
\end{tabular}


A.C. ADOMOU et al. / Int. J. Biol. Chem. Sci. 6(2): 745-772, 2012

\begin{tabular}{|c|c|c|c|c|c|c|c|}
\hline & & & & & & cutanées & \\
\hline $\begin{array}{l}\text { * Catharanthus } \\
\text { roseus }\end{array}$ & Bonjourbonsoirdo & Apocynaceae & nph & $\mathrm{Pt}$ & $\mathrm{Ra}$ & Hypertension & $\begin{array}{l}\text { Décoction } \\
\text { (or) }\end{array}$ \\
\hline Celosia trigyna & Adjikènu & Amaranthaceae & Th & PT & Tfe & $\begin{array}{l}\text { Accouchement } \\
\text { difficile }\end{array}$ & $\begin{array}{l}\text { Décoction } \\
\text { (or) }\end{array}$ \\
\hline $\begin{array}{l}\text { Chamaecrista } \\
\text { mimosoides }\end{array}$ & Atabouman & $\begin{array}{c}\text { Leg.- } \\
\text { Mimosoideae }\end{array}$ & Th & At & Tfe & Troubles de dentition & $\begin{array}{l}\text { Décoction } \\
\text { (or) }\end{array}$ \\
\hline Chassalia kolly & Djètiman & Rubiaceae & nph & SG & $\begin{array}{l}\text { Tfe- } \\
\mathrm{Ra}\end{array}$ & $\begin{array}{c}\text { Stérilité féminine, } \\
\text { Kyste, } \\
\text { fibrome,myome }\end{array}$ & $\begin{array}{l}\text { Décoction } \\
\text { (or) }\end{array}$ \\
\hline $\begin{array}{l}\text { * Chenopodium } \\
\text { ambrosioides }\end{array}$ & Amantrouzu, Gogo & Chenopodiaceae & Th & $\mathrm{Pt}$ & Tfe & $\begin{array}{c}\text { Infections, } \\
\text { vomissement }\end{array}$ & $\begin{array}{l}\text { Décoction } \\
\text { (or) }\end{array}$ \\
\hline $\begin{array}{l}\text { Cissampelos } \\
\text { owariensis }\end{array}$ & Djokodjè & Menispermaceae & Lnph & At & Tfe & $\begin{array}{l}\text { Folie, magique (porte } \\
\text { chance) }\end{array}$ & $\begin{array}{c}\text { Décoction } \\
\text { (or) et Savon } \\
\text { (bain) }\end{array}$ \\
\hline Cissus populnea & Dedo & Vitaceae & LHc & $\mathrm{S}$ & $\mathrm{Ra}$ & Faiblesse sexuelle & $\begin{array}{l}\text { Macération } \\
\text { (or) }\end{array}$ \\
\hline $\begin{array}{l}\text { Cissus } \\
\text { quadrangularis }\end{array}$ & Kankpé & Vitaceae & Lmph & $\mathrm{SZ}$ & $\mathrm{Tfe}$ & Fracture des os & $\begin{array}{l}\text { Décoction } \\
\text { (or) }\end{array}$ \\
\hline * Citrus limon & Clédo & Rutaceae & $\mathrm{mph}$ & $\mathrm{Pt}$ & $\mathrm{Ra}$ & Infections & $\begin{array}{l}\text { Décoction } \\
\text { (or) }\end{array}$ \\
\hline Clausena anisata & Gbozoazohion & Rutaceae & nph & $\mathrm{SG}$ & $\mathrm{Ra}$ & $\begin{array}{c}\text { Maux de ventre, } \\
\text { infections, diabète }\end{array}$ & $\begin{array}{l}\text { Décoction } \\
\text { (or) }\end{array}$ \\
\hline $\begin{array}{l}\text { Cleistopholis } \\
\text { patens }\end{array}$ & Hussokugoto & Annonaceae & $\mathrm{mPh}$ & GC & Ec & $\begin{array}{c}\text { Stérilité féminine, } \\
\text { kyste, fibrome,myome }\end{array}$ & $\begin{array}{l}\text { Décoction } \\
\text { (or) }\end{array}$ \\
\hline Cleome viscosa & Agbodokaya & Capparaceae & Th & At & Tfe & $\begin{array}{l}\text { Infections cutanées, } \\
\text { otites, paludisme, }\end{array}$ & $\begin{array}{l}\text { Décoction } \\
\text { (or) }\end{array}$ \\
\hline
\end{tabular}


A.C. ADOMOU et al. / Int. J. Biol. Chem. Sci. 6(2): 745-772, 2012

\begin{tabular}{|c|c|c|c|c|c|c|c|}
\hline & & & & & & ictère & \\
\hline $\begin{array}{l}\text { Clerodendrum } \\
\text { volubile }\end{array}$ & Adukowiwi & Verbenaceae & Lmph & $\mathrm{GC}$ & Tfe & Troubles de dentition & $\begin{array}{l}\text { Décoction } \\
\text { (or) }\end{array}$ \\
\hline $\begin{array}{l}\text { Cnestis } \\
\text { ferruginea }\end{array}$ & Gboviahu & Connaraceae & nph & SG & Tfe & Diarrhée & Savon (bain) \\
\hline * Cocos nucifera & Agonkèdo & Arecaceae & $\mathrm{mPh}$ & $\mathrm{Pt}$ & $\mathrm{Ra}$ & Anémie, ictère & $\begin{array}{l}\text { Décoction } \\
\text { (or) }\end{array}$ \\
\hline * Cola acuminata & $\mathrm{Vi}$ & Sterculiaceae & $\mathrm{mPh}$ & $\mathrm{GC}$ & $\begin{array}{l}\text { Gr- } \\
\mathrm{Ra}\end{array}$ & $\begin{array}{c}\text { Rougeole, } \\
\text { hypertension, stérilité } \\
\text { féminine }\end{array}$ & $\begin{array}{l}\text { Décoction } \\
\text { (or) }\end{array}$ \\
\hline Cola millenii & Aloviaton & Sterculiaceae & $\mathrm{mph}$ & $\mathrm{GC}$ & Tfe & Paludisme & $\begin{array}{l}\text { Décoction } \\
\text { (or) }\end{array}$ \\
\hline $\begin{array}{l}\text { Combretum } \\
\text { mucronatum }\end{array}$ & Adoussito & Combretaceae & Lnph & $\mathrm{GC}$ & Tfe & Diarrhée (enfants) & $\begin{array}{l}\text { Décoction } \\
\text { (or) }\end{array}$ \\
\hline $\begin{array}{l}\text { Combretum } \\
\text { paniculatum }\end{array}$ & Adanvèman & Combretaceae & Lnph & SG & Tfe & $\begin{array}{c}\text { Troubles menstruels, } \\
\text { stérilité féminine }\end{array}$ & $\begin{array}{c}\text { Décoction } \\
\text { (or) }\end{array}$ \\
\hline $\begin{array}{l}\text { Combretum } \\
\text { racemosum }\end{array}$ & Wèman & Combretaceae & Lmph & SG & Tfe & Infections & $\begin{array}{l}\text { Décoction } \\
\text { (or) }\end{array}$ \\
\hline $\begin{array}{l}\text { * Commiphora } \\
\text { africana }\end{array}$ & Lidjiman & Burseraceae & $\mathrm{mph}$ & $\mathrm{S}$ & Tfe & $\begin{array}{l}\text { Magique (porte } \\
\text { chance), } \\
\text { conjonctivites }\end{array}$ & Savon (bain) \\
\hline $\begin{array}{l}\text { * Copaifera } \\
\text { salikounda }\end{array}$ & Akpaflo & $\begin{array}{c}\text { Leg.- } \\
\text { Caesalpinioideae }\end{array}$ & $\mathrm{mPh}$ & $\mathrm{GC}$ & $\mathrm{Fr}$ & Infection & $\begin{array}{c}\text { Décoction } \\
\text { (or) }\end{array}$ \\
\hline Costus afer & Trétrégougou & Zingiberaceae & Gr & At & Tfe & Paludisme & $\begin{array}{l}\text { Décoction } \\
\text { (or) }\end{array}$ \\
\hline $\begin{array}{l}\text { Crateva } \\
\text { adansonii }\end{array}$ & Hontonzuzoué & Capparaceae & $\mathrm{mph}$ & PT & Tfe & Infections cutanées & $\begin{array}{l}\text { Décoction } \\
\text { (or) }\end{array}$ \\
\hline
\end{tabular}


A.C. ADOMOU et al. / Int. J. Biol. Chem. Sci. 6(2): 745-772, 2012

\begin{tabular}{|c|c|c|c|c|c|c|c|}
\hline Croton lobatus & Gbodudjogo & Euphorbiaceae & $\mathrm{Th}$ & At & Tfe & Infections cutanées & $\begin{array}{l}\text { Décoction } \\
\text { (or) }\end{array}$ \\
\hline $\begin{array}{l}\text { * Croton } \\
\text { zambesicus }\end{array}$ & Djélélé & Euphorbiaceae & $\mathrm{mPh}$ & $\mathrm{Pt}$ & $\begin{array}{l}\text { Tfe- } \\
\mathrm{Ra}\end{array}$ & $\begin{array}{c}\text { Infections cutanées- } \\
\text { magique }\end{array}$ & $\begin{array}{l}\text { Décoction } \\
\text { (or) }\end{array}$ \\
\hline $\begin{array}{l}\text { Cucumis melo } \\
\text { subsp. Agrestis }\end{array}$ & kablougoudou & Cucurbiatceae & Lnph & $\mathrm{Pt}$ & Tfe & $\begin{array}{l}\text { Kyste, myome, } \\
\text { fibrome }\end{array}$ & $\begin{array}{l}\text { Décoction } \\
\text { (or) }\end{array}$ \\
\hline $\begin{array}{l}\text { Cucumis } \\
\text { metuliferus }\end{array}$ & Gbohunon & Curcubitaceae & LTh & At & Fr & $\begin{array}{c}\text { Rougeole (maladies } \\
\text { éruptives) }\end{array}$ & $\begin{array}{l}\text { Décoction } \\
\text { (or) }\end{array}$ \\
\hline Curculigo pilosa & Ayoté & Hypoxidaceae & Gt & At & $\mathrm{Rh}$ & Infections & $\begin{array}{l}\text { Décoction } \\
\text { (or) }\end{array}$ \\
\hline $\begin{array}{l}\text { * Cynometra } \\
\text { megalophylla }\end{array}$ & Bougoto & $\begin{array}{c}\text { Leg.- } \\
\text { Caesalpinioideae }\end{array}$ & $\mathrm{mPh}$ & $\mathrm{GC}$ & $\mathrm{Ec}$ & Anémie & $\begin{array}{c}\text { Décoction } \\
\text { (or) }\end{array}$ \\
\hline $\begin{array}{l}\text { Cyperus } \\
\text { esculentus }\end{array}$ & Afiyo & Cyperaceae & $\mathrm{Gr}$ & $\mathrm{Pt}$ & $\mathrm{Rh}$ & Faiblesse sexuelle & $\begin{array}{l}\text { Décoction } \\
\text { (or) }\end{array}$ \\
\hline $\begin{array}{l}\text { Desmodium } \\
\text { ramossissimum }\end{array}$ & Zèdali & $\begin{array}{c}\text { Leg.- } \\
\text { Papilionoideae }\end{array}$ & Th & $\mathrm{Pt}$ & Tfe & $\begin{array}{l}\text { Accouchement } \\
\text { difficile }\end{array}$ & $\begin{array}{l}\text { Décoction } \\
\text { (or) }\end{array}$ \\
\hline $\begin{array}{l}\text { Dialium } \\
\text { guineense }\end{array}$ & Assouinssouinman & $\begin{array}{c}\text { Leg.- } \\
\text { Caesalpinioideae }\end{array}$ & $\mathrm{mPh}$ & SG & Tfe & Paludisme & $\begin{array}{c}\text { Décoction } \\
\text { (or) }\end{array}$ \\
\hline $\begin{array}{l}\text { Dichapetalum } \\
\text { madagascariense }\end{array}$ & Gbaglo & Dichapetalaceae & $\mathrm{mph}$ & SG & Tfe & Paludisme & $\begin{array}{l}\text { Décoction } \\
\text { (or) }\end{array}$ \\
\hline $\begin{array}{l}\text { Dichrostachys } \\
\text { cinerea }\end{array}$ & Badawin & $\begin{array}{c}\text { Leg.- } \\
\text { Mimosoideae }\end{array}$ & nph & SG & $\begin{array}{c}\text { Tfe- } \\
\mathrm{Ra}\end{array}$ & $\begin{array}{c}\text { Rougeole, Hernie, } \\
\text { ulcère }\end{array}$ & $\begin{array}{c}\text { Décoction } \\
\text { (or) }\end{array}$ \\
\hline $\begin{array}{l}\text { Dicoma } \\
\text { tomentosa }\end{array}$ & Ahéhé & Asteraceae & $\mathrm{Th}$ & $\mathrm{Pt}$ & Tfe & $\begin{array}{l}\text { Magique (porte } \\
\text { chance) }\end{array}$ & Savon (bain) \\
\hline Diodia scandens & Sèhoin & Rubiaceae & $\mathrm{Th}$ & $\mathrm{Pt}$ & Tfe & $\begin{array}{c}\text { Troubles de miction } \\
\text { (femme enceinte) }\end{array}$ & $\begin{array}{l}\text { Décoction } \\
\text { (or) }\end{array}$ \\
\hline Ehretia cymosa & Mignonman & Boraginaceae & $\mathrm{mph}$ & $\mathrm{SG}$ & $\mathrm{Tfe}$ & Fièvre & Décoction \\
\hline
\end{tabular}


A.C. ADOMOU et al. / Int. J. Biol. Chem. Sci. 6(2): 745-772, 2012

\begin{tabular}{|c|c|c|c|c|c|c|c|}
\hline & & & & & & & (or) \\
\hline $\begin{array}{l}\text { Emilia } \\
\text { praetermissa }\end{array}$ & Amoudimoudi & Asteraceae & $\mathrm{Th}$ & SG & $\mathrm{Tfe}$ & $\begin{array}{l}\text { Magique (porte } \\
\text { chance) }\end{array}$ & Savon (bain) \\
\hline Entada gigas & Gbagbala & $\begin{array}{c}\text { Leg.- } \\
\text { Mimosoideae }\end{array}$ & $\mathrm{LmPh}$ & $\mathrm{GC}$ & Gr & Infection & $\begin{array}{l}\text { Décoction } \\
\text { (or) }\end{array}$ \\
\hline $\begin{array}{l}\text { Eriosema } \\
\text { glomerata }\end{array}$ & Adjahiman & $\begin{array}{c}\text { Leg.- } \\
\text { Papilionoideae }\end{array}$ & $\mathrm{Ch}$ & SG & Tfe & $\begin{array}{c}\text { Fragilité de la } \\
\text { fontanelle (nouveau- } \\
\text { né) }\end{array}$ & $\begin{array}{l}\text { Décoction } \\
\text { (or) }\end{array}$ \\
\hline $\begin{array}{l}\text { Eugenia } \\
\text { aromatica }\end{array}$ & Atikingbadota & Myrtaceae & $\mathrm{mph}$ & $\mathrm{Pt}$ & $\mathrm{Gr}$ & Infection & $\begin{array}{c}\text { Décoction } \\
\text { (or) }\end{array}$ \\
\hline Eugenia sp & Plèplè & Myrtaceae & $?$ & $?$ & $\mathrm{Gr}$ & Faiblesse sexuelle & $\begin{array}{l}\text { Décoction } \\
\text { (or) }\end{array}$ \\
\hline $\begin{array}{l}\text { * Euphorbia } \\
\text { kamerunica }\end{array}$ & Lanmadou sélo & Euphorbiaceae & $\mathrm{mph}$ & $\mathrm{GC}$ & Tfe & $\begin{array}{c}\text { Infections virales } \\
(\mathrm{VIH})\end{array}$ & $\begin{array}{l}\text { Décoction } \\
\text { (or) }\end{array}$ \\
\hline $\begin{array}{l}\text { Evolvulus } \\
\text { alsinioides }\end{array}$ & Droman & Convolulaceae & $\mathrm{Ch}$ & $\mathrm{Pt}$ & Tfe & $\begin{array}{c}\text { Cauchemars, } \\
\text { convulsions (enfants) }\end{array}$ & $\begin{array}{l}\text { Décoction } \\
\text { (or) }\end{array}$ \\
\hline Ficus exasperata & Aholoman & Moraceae & $\mathrm{mPh}$ & $\mathrm{GC}$ & $\mathrm{Tfe}$ & $\begin{array}{l}\text { Surpoids chez le fœtus } \\
\text { (grossesse) }\end{array}$ & $\begin{array}{l}\text { Décoction } \\
\text { (or) }\end{array}$ \\
\hline Flacourtia indica & Gbohoukadjè & Flacourtiaceae & $\mathrm{mph}$ & At & $\begin{array}{c}\text { Tfe- } \\
\mathrm{Ra}\end{array}$ & Paludisme-Anémie & $\begin{array}{c}\text { Décoction } \\
\text { (or) }\end{array}$ \\
\hline Flueggea virosa & Tchakètchakè & Euphorbiaceae & nph & $\mathrm{Pt}$ & $\mathrm{Tfe}$ & Paludisme & $\begin{array}{l}\text { Décoction } \\
\text { (or) }\end{array}$ \\
\hline * Garcinia kola & Ahoyé & Clusiaceae & $\mathrm{mPh}$ & $\mathrm{GC}$ & $\begin{array}{l}\mathrm{Ra}, \\
\mathrm{Gr}, \\
\text { éc }\end{array}$ & $\begin{array}{l}\text { Faiblesse sexuelle, } \\
\text { toux }\end{array}$ & $\begin{array}{l}\text { Décoction } \\
\text { (or) }\end{array}$ \\
\hline $\begin{array}{l}\text { Gardenia } \\
\text { erubescens }\end{array}$ & Dakplado & Rubiaceae & nph & $S$ & $\mathrm{Ra}$ & Hypertension & $\begin{array}{l}\text { Décoction } \\
\text { (or) }\end{array}$ \\
\hline
\end{tabular}


A.C. ADOMOU et al. / Int. J. Biol. Chem. Sci. 6(2): 745-772, 2012

\begin{tabular}{|c|c|c|c|c|c|c|c|}
\hline $\begin{array}{c}\text { Gardenia } \\
\text { ternifolia }\end{array}$ & Dakplado & Rubiaceae & nph & SZ & $\mathrm{Ra}$ & Hypertension & $\begin{array}{l}\text { Décoction } \\
\text { (or) }\end{array}$ \\
\hline Gladiolus dalenii & Baka & Iridaceae & $\mathrm{Gb}$ & At & $\mathrm{Rh}$ & Infection & $\begin{array}{l}\text { Décoction } \\
\text { (or) }\end{array}$ \\
\hline $\begin{array}{l}\text { Glinus } \\
\text { opppositifolius }\end{array}$ & Wanichè & Molluginaceae & Th & $\mathrm{Pt}$ & Tfe & $\begin{array}{l}\text { Magique (porte } \\
\text { chance) }\end{array}$ & Savon (bain) \\
\hline $\begin{array}{l}\text { Gomphrena } \\
\text { celosioides }\end{array}$ & Adukowé & Amaranthaceae & Th & $\mathrm{Pt}$ & Tfe & $\begin{array}{l}\text { Troubles de dentition } \\
\text { (enfants) }\end{array}$ & $\begin{array}{l}\text { Décoction } \\
\text { (or) }\end{array}$ \\
\hline Gossypium spp & Cotoman & Malvaceae & $\mathrm{nph}$ & $\mathrm{Pt}$ & Tfe & Paludisme & $\begin{array}{l}\text { Décoction } \\
\text { (or) }\end{array}$ \\
\hline $\begin{array}{l}\text { Harissonia } \\
\text { abyssinica }\end{array}$ & Kpatikikonman & Simaroubaceae & $\mathrm{mph}$ & SG & Tfe & Infections cutanées & $\begin{array}{l}\text { Décoction } \\
\text { (or) }\end{array}$ \\
\hline $\begin{array}{l}\text { * Heliotropium } \\
\text { indicum }\end{array}$ & Koclossoudinkpatcha & Boraginaceae & Th & SG & Tfe & $\begin{array}{l}\text { Hypertension, } \\
\text { Infections }\end{array}$ & $\begin{array}{l}\text { Décoction } \\
\text { (or) }\end{array}$ \\
\hline $\begin{array}{l}\text { Heterotis } \\
\text { rotundifolia }\end{array}$ & Hèhè & Melastomataceae & Th & At & Tfe & $\begin{array}{c}\text { Troubles de miction } \\
\text { chez la femme } \\
\text { enceinte }\end{array}$ & $\begin{array}{l}\text { Décoction } \\
\text { (or) }\end{array}$ \\
\hline $\begin{array}{l}\text { * Hibiscus } \\
\text { acetolosa }\end{array}$ & Gissiman & Malvaceae & nph & $\mathrm{Pt}$ & Tfe & Paludisme & Savon (bain) \\
\hline $\begin{array}{l}\text { Hibiscus } \\
\text { surrattensis }\end{array}$ & Kpofin & Malvaceae & nph & $\mathrm{Pt}$ & Tfe & Paludisme & $\begin{array}{c}\text { Décoction } \\
\text { (or) }\end{array}$ \\
\hline $\begin{array}{l}\text { Hoslundia } \\
\text { opposita }\end{array}$ & Agahouman & Lamiaceae & $\mathrm{nph}$ & $\mathrm{Pt}$ & Tfe & Infections & $\begin{array}{l}\text { Décoction } \\
\text { (or) }\end{array}$ \\
\hline $\begin{array}{l}\text { Hybanthus } \\
\text { enneaspermus }\end{array}$ & Abiwèlè & Violaceae & Th & $\mathrm{Pt}$ & Tfe & $\begin{array}{l}\text { Accouchement } \\
\text { difficile }\end{array}$ & $\begin{array}{l}\text { Décoction } \\
\text { (or) }\end{array}$ \\
\hline $\begin{array}{l}\text { Hymenocardia } \\
\text { acida }\end{array}$ & faux Adjassiafovè & Euphorbiaceae & $\mathrm{mph}$ & SZ & Tfe & Infections & $\begin{array}{l}\text { Décoction } \\
\text { (or) }\end{array}$ \\
\hline
\end{tabular}


A.C. ADOMOU et al. / Int. J. Biol. Chem. Sci. 6(2): 745-772, 2012

\begin{tabular}{|c|c|c|c|c|c|c|c|}
\hline $\begin{array}{l}\text { Hyptis } \\
\text { Suaveolens }\end{array}$ & Afio & Lamiaceae & Th & $\mathrm{Pt}$ & Tfe & $\begin{array}{c}\text { Infections et } \\
\text { démangeaison } \\
\text { cutanées }\end{array}$ & $\begin{array}{l}\text { Décoction } \\
\text { (or) }\end{array}$ \\
\hline $\begin{array}{l}\text { Indigofera } \\
\text { pulchra }\end{array}$ & Zuko ou Koma & $\begin{array}{c}\text { Leg.- } \\
\text { Papilionoideae }\end{array}$ & $\mathrm{Ch}$ & SG & Tfe & $\begin{array}{c}\text { Retard staturo- } \\
\text { pondéral (enfants) }\end{array}$ & $\begin{array}{c}\text { Décoction } \\
\text { (or) }\end{array}$ \\
\hline $\begin{array}{l}\text { * Justicia } \\
\text { secunda }\end{array}$ & Houmanssi & Acanthaceae & Th & $\mathrm{Pt}$ & Tfe & Anémie & $\begin{array}{c}\text { Décoction } \\
\text { (or) }\end{array}$ \\
\hline $\begin{array}{l}\text { * Kalankoe } \\
\text { crenata }\end{array}$ & Afaman djomakou & Crassulaceae & nph & SG & Tfe & Toux & $\begin{array}{l}\text { Décoction } \\
\text { (or) }\end{array}$ \\
\hline $\begin{array}{l}\text { Kedrostis } \\
\text { foetidissima }\end{array}$ & Chiyoman & Cucurbiatceae & Lnph & At & Tfe & $\begin{array}{l}\text { Magique (porte } \\
\text { chance) }\end{array}$ & Savon (bain) \\
\hline Keetia hispida & Adjadjin & Rubiaceae & Lmph & GC & Tfe & $\begin{array}{c}\text { Retard staturo- } \\
\text { pondéral (enfants) }\end{array}$ & $\begin{array}{l}\text { Décoction } \\
\text { (or) }\end{array}$ \\
\hline $\begin{array}{l}\text { * Khaya } \\
\text { senegalensis }\end{array}$ & Kaïlcédra & Meliaceae & $\mathrm{mPh}$ & S & Tfe & Infections & $\begin{array}{l}\text { Décoction } \\
\text { (or) }\end{array}$ \\
\hline Kigelia africana & Gnanblikpo & Bignoniaceae & $\mathrm{mPh}$ & SG & $\begin{array}{l}\text { Fr- } \\
\text { Ec }\end{array}$ & $\begin{array}{l}\text { Infection, kyste, } \\
\text { myome, fibrome }\end{array}$ & $\begin{array}{l}\text { Décoction } \\
\text { (or) }\end{array}$ \\
\hline $\begin{array}{l}\text { * Lagenaria } \\
\text { siceraria }\end{array}$ & Goussi & Cucurbitaceae & Th & $\mathrm{Pt}$ & Fr & Infections & $\begin{array}{c}\text { Décoction } \\
\text { (or) }\end{array}$ \\
\hline Lannea barteri & Zuzugoto & Anacardiaceae & $\mathrm{mPh}$ & $\mathrm{S}$ & Ec & Anémie & $\begin{array}{l}\text { Décoction } \\
\text { (or) }\end{array}$ \\
\hline $\begin{array}{l}\text { * Lantana } \\
\text { camara }\end{array}$ & Hlachiayo & Verbenaceae & Lmph & SG & Tfe & Infections & $\begin{array}{l}\text { Décoction } \\
\text { (or) }\end{array}$ \\
\hline $\begin{array}{l}\text { Launaea } \\
\text { taraxacifolia }\end{array}$ & Gnantotéyé & Asteraceae & Th & S & Tfe & $\begin{array}{l}\text { Magique (porte } \\
\text { chance) }\end{array}$ & Savon (bain) \\
\hline $\begin{array}{l}\text { Lecaniodiscus } \\
\text { cupanioides }\end{array}$ & Ganhotido & Sapindaceae & mph & GC & $\mathrm{Ra}$ & $\begin{array}{c}\text { Kyste, } \\
\text { fibrome,myome }\end{array}$ & $\begin{array}{l}\text { Décoction } \\
\text { (or) }\end{array}$ \\
\hline
\end{tabular}


A.C. ADOMOU et al. / Int. J. Biol. Chem. Sci. 6(2): 745-772, 2012

\begin{tabular}{|c|c|c|c|c|c|c|c|}
\hline $\begin{array}{l}\text { Leucas } \\
\text { martinicensis }\end{array}$ & Adoutonwé & Lamiaceae & Th & $\mathrm{Pt}$ & Tfe & $\begin{array}{c}\text { Troubles de dentition } \\
\text { (enfants) }\end{array}$ & $\begin{array}{l}\text { Décoction } \\
\text { (or) }\end{array}$ \\
\hline Lippia multiflora & Agalala & Verbenaceae & nph & SG & Tfe & $\begin{array}{l}\text { Infections cutanées, } \\
\text { hypertension }\end{array}$ & $\begin{array}{l}\text { Décoction } \\
\text { (or) }\end{array}$ \\
\hline $\begin{array}{l}\text { Lonchocarpus } \\
\text { sericeus }\end{array}$ & Nombala & $\begin{array}{c}\text { Leg.- } \\
\text { Papilionoideae }\end{array}$ & $\mathrm{mph}$ & SG & Tfe & $\begin{array}{l}\text { Constipation } \\
\text { (purgatif) }\end{array}$ & $\begin{array}{l}\text { Décoction } \\
\text { (or) }\end{array}$ \\
\hline $\begin{array}{l}\text { Lycopodiella } \\
\text { cernua }\end{array}$ & Hingblé & Lycopodiaceae & Lnph & At & Tfe & $\begin{array}{l}\text { Magique (Porte } \\
\text { chance) }\end{array}$ & Savon (bain) \\
\hline $\begin{array}{l}\text { Macrosphyra } \\
\text { longistyla }\end{array}$ & Chibidigohu & Rubiaceae & Lnph & SG & Tfe & Infections & Savon (bain) \\
\hline $\begin{array}{l}* \text { Mangifera } \\
\text { indica }\end{array}$ & Amangagoto & Anacardiaceae & $\mathrm{mPh}$ & $\mathrm{Pt}$ & $\mathrm{Ra}$ & Anémie, toux & $\begin{array}{l}\text { Décoction } \\
\text { (or) }\end{array}$ \\
\hline $\begin{array}{l}\text { Manilkara } \\
\text { multinervis }\end{array}$ & Adjiman & Sapotaceae & $\mathrm{mph}$ & SG & Tfe & $\begin{array}{c}\text { Inhalation du liquide } \\
\text { amniotique par le } \\
\text { nouveau-né }\end{array}$ & $\begin{array}{l}\text { Décoction } \\
\text { (or) }\end{array}$ \\
\hline $\begin{array}{l}\text { * Melaleuca } \\
\text { leucadendron }\end{array}$ & $\begin{array}{l}\text { Kpinmanssi } \\
\text { Sèminton }\end{array}$ & Myrtaceae & $\mathrm{mPh}$ & $\mathrm{Pt}$ & Tfe & Toux & $\begin{array}{l}\text { Décoction } \\
\text { (or) }\end{array}$ \\
\hline $\begin{array}{l}\text { Merremia } \\
\text { tridentata }\end{array}$ & Honhou & Convolulaceae & Lnph & $\mathrm{Pt}$ & Tfe & $\begin{array}{c}\text { Accouchement } \\
\text { difficile }\end{array}$ & $\begin{array}{c}\text { Décoction } \\
\text { (or) }\end{array}$ \\
\hline $\begin{array}{l}\text { Microsorum } \\
\text { scolopendria }\end{array}$ & Degoman & Polypodiaceae & Gr & $\mathrm{Pt}$ & Tfe & Paludisme & $\begin{array}{l}\text { Décoction } \\
\text { (or) }\end{array}$ \\
\hline $\begin{array}{l}\text { Mitracarpus } \\
\text { hirtus }\end{array}$ & Godoko & Rubiaceae & Th & $\mathrm{Pt}$ & Tfe & Dermatoses & $\begin{array}{l}\text { Décoction } \\
\text { (or) }\end{array}$ \\
\hline $\begin{array}{l}\text { Mollugo } \\
\text { nudicaulis }\end{array}$ & Vèni & Molluginaceae & Th & At & Tfe & $\begin{array}{l}\text { Magique (porte } \\
\text { chance) }\end{array}$ & Savon (bain) \\
\hline Mondia whitei & Chriligoun & Asclepiadaceae & $\mathrm{mPh}$ & At & $\mathrm{Ra}$ & Faiblesse sexuelle & $\begin{array}{c}\text { Décoction } \\
\text { (or) }\end{array}$ \\
\hline
\end{tabular}


A.C. ADOMOU et al. / Int. J. Biol. Chem. Sci. 6(2): 745-772, 2012

\begin{tabular}{|c|c|c|c|c|c|c|c|}
\hline $\begin{array}{l}\text { Monodora } \\
\text { myristica }\end{array}$ & Sasaliku & Annonaceae & $\mathrm{mPh}$ & GC & $\begin{array}{l}\text { Fr- } \\
\mathrm{Ra}\end{array}$ & $\begin{array}{l}\text { Infection-Kyste, } \\
\text { fibrome,myome }\end{array}$ & $\begin{array}{l}\text { Décoction } \\
\text { (or) }\end{array}$ \\
\hline Morinda lucida & Houinssin & Rubiaceae & $\mathrm{mPh}$ & $\mathrm{Pt}$ & $\begin{array}{l}\text { Tfe- } \\
\mathrm{Ra}\end{array}$ & $\begin{array}{c}\text { Troubles menstruels, } \\
\text { stérilité-diabète, } \\
\text { troubles menstruels, } \\
\text { anémie }\end{array}$ & $\begin{array}{l}\text { Décoction } \\
\text { (or) }\end{array}$ \\
\hline $\begin{array}{l}\text { Nauclea } \\
\text { diderrichii }\end{array}$ & Atahégoto & Rubiaceae & $\mathrm{mPh}$ & $\mathrm{GC}$ & Ec & Ictère, paludisme & $\begin{array}{c}\text { Décoction } \\
\text { (or) }\end{array}$ \\
\hline $\begin{array}{l}\text { * Newbouldia } \\
\text { leavis }\end{array}$ & Dessléguè & Bignoniaceae & $\mathrm{mph}$ & $\mathrm{SG}$ & $\mathrm{Tfe}$ & $\begin{array}{c}\text { Accouchement } \\
\text { difficile, magique }\end{array}$ & $\begin{array}{l}\text { Décoction } \\
\text { (or) }\end{array}$ \\
\hline * Ocimum canum & Késsoukéssou & Lamiaceae & $\mathrm{Ch}$ & $\mathrm{Pt}$ & Tfe & Infections & $\begin{array}{l}\text { Décoction } \\
\text { (or) }\end{array}$ \\
\hline $\begin{array}{l}\text { * Ocimum } \\
\text { gratissimum }\end{array}$ & Tchiayo & Lamiaceae & $\mathrm{Ch}$ & At & $\mathrm{Tfe}$ & Infections & $\begin{array}{l}\text { Décoction } \\
\text { (or) }\end{array}$ \\
\hline $\begin{array}{l}\text { Olax } \\
\text { subscorpiö̈dea }\end{array}$ & Amitoudo & Olacaceae & $\mathrm{mph}$ & $\mathrm{GC}$ & $\mathrm{Ra}$ & $\begin{array}{c}\text { Kyste, } \\
\text { fibrome,myome }\end{array}$ & $\begin{array}{l}\text { Décoction } \\
\text { (or) }\end{array}$ \\
\hline Opilia amentacea & Tonahantondo & Opiliaceae & $\mathrm{mph}$ & $\mathrm{SZ}$ & $\mathrm{Ra}$ & Maux de tête & $\begin{array}{l}\text { Décoction } \\
\text { (or) }\end{array}$ \\
\hline $\begin{array}{l}\text { Pancratium } \\
\text { tenuifolium }\end{array}$ & Ganganyonlon & Amaranthaceae & $\mathrm{Gb}$ & S & $\mathrm{Bu}$ & Toux & $\begin{array}{l}\text { Décoction } \\
\text { (or) }\end{array}$ \\
\hline $\begin{array}{l}\text { Pandiaka } \\
\text { involucrata }\end{array}$ & Vrai adjassiafovè & Amaranthaceae & $\mathrm{Ch}$ & $S$ & Tfe & Infections cutanées & $\begin{array}{l}\text { Décoction } \\
\text { (or) }\end{array}$ \\
\hline Parkia biglobosa & Ahagoto & $\begin{array}{c}\text { Leg.- } \\
\text { Mimosoideae }\end{array}$ & $\mathrm{mPh}$ & $S$ & $\mathrm{Ra}$ & $\begin{array}{l}\text { Hypertension, Retard } \\
\text { staturo-pondéral } \\
\text { (bébés) }\end{array}$ & $\begin{array}{l}\text { Décoction } \\
\text { (or) }\end{array}$ \\
\hline Passiflora foetida & Avugnintru & Passifloraceae & Lnph & $\mathrm{Pt}$ & $\mathrm{Tfe}$ & Dermatoses (bébés) & $\begin{array}{l}\text { Décoction } \\
\text { (or) }\end{array}$ \\
\hline
\end{tabular}


A.C. ADOMOU et al. / Int. J. Biol. Chem. Sci. 6(2): 745-772, 2012

\begin{tabular}{|c|c|c|c|c|c|c|c|}
\hline Paullinia pinnata & Hèdulifi & Sapindaceae & Lmph & At & $\mathrm{Tfe}$ & $\begin{array}{c}\text { Troubles de la } \\
\text { dentition, stérilité }\end{array}$ & $\begin{array}{l}\text { Décoction } \\
\text { (or) }\end{array}$ \\
\hline $\begin{array}{l}\text { Pauridiantha } \\
\text { hirtella }\end{array}$ & Towé & Rubiaceae & $\mathrm{mph}$ & $\mathrm{GC}$ & $\mathrm{Tfe}$ & Toux (bébés) & $\begin{array}{l}\text { Décoction } \\
\quad \text { (or) }\end{array}$ \\
\hline $\begin{array}{l}\text { Pavetta } \\
\text { corymbosa }\end{array}$ & Lokou & Rubiaceae & $\mathrm{mph}$ & SG & $\mathrm{Tfe}$ & Paludisme & $\begin{array}{l}\text { Décoction } \\
\quad \text { (or) }\end{array}$ \\
\hline Pavetta crassipes & Parakouman & Rubiaceae & nph & At & $\mathrm{Tfe}$ & Paludisme & $\begin{array}{l}\text { Décoction } \\
\text { (or) }\end{array}$ \\
\hline $\begin{array}{l}\text { Pennisetum } \\
\text { purpureum }\end{array}$ & Fan vovo & Poaceae & $\mathrm{Th}$ & At & $\mathrm{Tfe}$ & Anémie & $\begin{array}{l}\text { Décoction } \\
\quad \text { (or) }\end{array}$ \\
\hline $\begin{array}{l}\text { Peperomia } \\
\text { pellucida }\end{array}$ & Fifaman & Piperaceae & $\mathrm{Th}$ & $\mathrm{Pt}$ & $\mathrm{Tfe}$ & $\begin{array}{l}\text { Stérilités, magique } \\
\text { (Porte chance) }\end{array}$ & $\begin{array}{c}\text { Décoction } \\
\text { (or) et Savon } \\
\text { (bain) }\end{array}$ \\
\hline $\begin{array}{l}\text { Pergularia } \\
\text { daemia }\end{array}$ & Kpinto & Asclepiadaceae & Lmph & SG & $\mathrm{Tfe}$ & Toux & $\begin{array}{l}\text { Décoction } \\
\text { (or) }\end{array}$ \\
\hline $\begin{array}{l}\text { Pericopsis } \\
\text { laxiflora }\end{array}$ & Sandju & $\begin{array}{c}\text { Leg.- } \\
\text { Papilionoideae } \\
\end{array}$ & $\mathrm{mph}$ & S & $\mathrm{Tfe}$ & $\begin{array}{l}\text { Magique (porte } \\
\text { chance) }\end{array}$ & $\begin{array}{l}\text { Décoction } \\
\text { (or) }\end{array}$ \\
\hline $\begin{array}{l}\text { Phaulopsis } \\
\text { ciliata }\end{array}$ & Chouchouglouchou & Acanthaceae & nph & SG & $\mathrm{Tfe}$ & Protection & Savon (bain) \\
\hline $\begin{array}{l}\text { Phyllanthus } \\
\text { amarus }\end{array}$ & Hlenwé & Euphorbiaceae & $\mathrm{Th}$ & $\mathrm{Pt}$ & $\mathrm{Tfe}$ & Paludisme & $\begin{array}{l}\text { Décoction } \\
\quad \text { (or) }\end{array}$ \\
\hline Physalis angulata & Issiman & Solanaceae & $\mathrm{Th}$ & $\mathrm{Pt}$ & $\mathrm{Tfe}$ & Paludisme & $\begin{array}{l}\text { Décoction } \\
\quad(\text { or })\end{array}$ \\
\hline $\begin{array}{l}\text { * Picralima } \\
\text { nitida }\end{array}$ & Ayokpè & Apocynaceae & $\mathrm{mph}$ & GC & $\mathrm{Fr}$ & Toux, angine & $\begin{array}{l}\text { Sucer la } \\
\text { graine }\end{array}$ \\
\hline * Piper guineense & Linlinku & Piperaceae & $\mathrm{LmPh}$ & GC & $\mathrm{Gr}$ & $\begin{array}{l}\text { Kyste, myome et } \\
\text { fibrome }\end{array}$ & $\begin{array}{l}\text { Décoction } \\
\text { (or) }\end{array}$ \\
\hline
\end{tabular}


A.C. ADOMOU et al. / Int. J. Biol. Chem. Sci. 6(2): 745-772, 2012

\begin{tabular}{|c|c|c|c|c|c|c|c|}
\hline $\begin{array}{l}\text { Pleiocarpa } \\
\text { pycnantha }\end{array}$ & Vonman & Apocynaceae & nph & At & Tfe & Vers intestinaux & $\begin{array}{l}\text { Décoction } \\
\text { (or) }\end{array}$ \\
\hline $\begin{array}{l}\text { * Plumbago } \\
\text { zeylanica }\end{array}$ & Dangblando & Plumbaginaceae & Lnph & $\mathrm{Pt}$ & $\mathrm{Ra}$ & $\begin{array}{c}\text { Hernie, Kyste, } \\
\text { fibrome, myome }\end{array}$ & $\begin{array}{l}\text { Décoction } \\
\text { (or) }\end{array}$ \\
\hline $\begin{array}{l}\text { Polycarpaea } \\
\text { linearifolia }\end{array}$ & Aduko & Caryophyllaceae & Th & $\mathrm{SZ}$ & Tfe & Troubles de dentition & $\begin{array}{l}\text { Décoction } \\
\text { (or) }\end{array}$ \\
\hline $\begin{array}{l}\text { Polygala } \\
\text { arenaria }\end{array}$ & Mili & Polygalaceae & Th & SG & Tfe & Troubles hépatiques & $\begin{array}{c}\text { Décoction } \\
\text { (or) }\end{array}$ \\
\hline Prosopis africana & Kakè & $\begin{array}{c}\text { Leg.- } \\
\text { Mimosoideae }\end{array}$ & $\mathrm{mPh}$ & $\mathrm{S}$ & $\operatorname{Tr}$ & $\begin{array}{c}\text { Retard staturo- } \\
\text { pondéral (enfants) }\end{array}$ & $\begin{array}{l}\text { Décoction } \\
\text { (bain) }\end{array}$ \\
\hline $\begin{array}{l}\text { Pseudocedrela } \\
\text { kotschyi }\end{array}$ & Atidohoukpédo & Meliaceae & $\mathrm{mph}$ & $\mathrm{S}$ & $\mathrm{Ra}$ & $\begin{array}{c}\text { Maux de ventre, } \\
\text { paludisme, infections, } \\
\text { diabète }\end{array}$ & $\begin{array}{l}\text { Décoction } \\
\text { (or) }\end{array}$ \\
\hline $\begin{array}{l}\text { * Psidium } \\
\text { guajava }\end{array}$ & Kinkounma & Myrtaceae & $\mathrm{mPh}$ & $\mathrm{Pt}$ & Tfe & Diarrhée & $\begin{array}{l}\text { Décoction } \\
\text { (or) }\end{array}$ \\
\hline $\begin{array}{l}\text { Psorospermum } \\
\text { glaberrimum }\end{array}$ & Mlanmi & Clusiaceae & $\mathrm{mph}$ & SG & Tfe & Infections cutanées & $\begin{array}{l}\text { Décoction } \\
\text { (or) }\end{array}$ \\
\hline $\begin{array}{l}\text { Psychotria } \\
\text { vogeliana }\end{array}$ & Démlango & Rubiaceae & nph & SG & Tfe & $\begin{array}{c}\text { Encombrement des } \\
\text { voies respiratoires } \\
\text { (bébé) }\end{array}$ & Savon (bain) \\
\hline $\begin{array}{l}\text { Pteleopsis } \\
\text { suberosa }\end{array}$ & kuilikuiligoto & Combretaceae & $\mathrm{mph}$ & SZ & $\mathrm{Ec}$ & Infections cutanées & $\begin{array}{l}\text { Décoction } \\
\text { (or) }\end{array}$ \\
\hline $\begin{array}{l}\text { Pterocarpus } \\
\text { erinaceus }\end{array}$ & Kossogoto & $\begin{array}{c}\text { Leg.- } \\
\text { Papilionoideae }\end{array}$ & $\mathrm{mPh}$ & $\mathrm{S}$ & $\mathrm{Ec}$ & $\begin{array}{c}\text { Anémie, troubles } \\
\text { menstruel }\end{array}$ & $\begin{array}{l}\text { Décoction } \\
\text { (or) }\end{array}$ \\
\hline $\begin{array}{l}\text { * Pterocarpus } \\
\text { santalinoides }\end{array}$ & Gbèougbèou & $\begin{array}{c}\text { Leg.- } \\
\text { Papilionoideae }\end{array}$ & $\mathrm{mph}$ & SG & Tfe & Diarrhée & $\begin{array}{l}\text { Décoction } \\
\text { (or) }\end{array}$ \\
\hline Pupalia lappacea & Trèdoavohou & Amaranthaceae & $\mathrm{Ch}$ & $\mathrm{Pt}$ & Tfe & Ictère, paludisme & Décoction \\
\hline
\end{tabular}


A.C. ADOMOU et al. / Int. J. Biol. Chem. Sci. 6(2): 745-772, 2012

\begin{tabular}{|c|c|c|c|c|c|c|c|}
\hline & & & & & & & (or) \\
\hline $\begin{array}{l}\text { Rauvolfia } \\
\text { vomitoria }\end{array}$ & Lètiwé & Apocynaceae & $\mathrm{mph}$ & SG & $\begin{array}{l}\text { Tfe- } \\
\mathrm{Ra}\end{array}$ & Folie & $\begin{array}{l}\text { Décoction } \\
\text { (or) }\end{array}$ \\
\hline $\begin{array}{l}\text { Remirea } \\
\text { maritima }\end{array}$ & Xuso & Melastomataceae & $\mathrm{Gr}$ & $\mathrm{Pt}$ & Tfe & Trouble de dentition & $\begin{array}{l}\text { Décoction } \\
\text { (or) }\end{array}$ \\
\hline $\begin{array}{l}\text { Rhaphiostylis } \\
\text { beninensis }\end{array}$ & Kplakplakan & Icacinaceae & Lnph & GC & Tige & $\begin{array}{c}\text { Infections, troubles } \\
\text { hépatiques, maux de } \\
\text { ventre }\end{array}$ & $\begin{array}{l}\text { Décoction } \\
\text { (or) }\end{array}$ \\
\hline $\begin{array}{l}* \\
\text { Rhodognaphalon } \\
\text { brevicuspe }\end{array}$ & kpatidèhï & Bombacaceae & $\mathrm{mPh}$ & GC & Tfe & Stérilité féminine & $\begin{array}{l}\text { Décoction } \\
\text { (or) }\end{array}$ \\
\hline Rourea coccinea & Vikplomba & Connaraceae & $\mathrm{nph}$ & $\mathrm{SG}$ & Tfe & Stérilité féminine & $\begin{array}{l}\text { Décoction } \\
\text { (or) }\end{array}$ \\
\hline $\begin{array}{l}\text { Rytigynia } \\
\text { umbellulata }\end{array}$ & Gbadéman & Rubiaceae & $\mathrm{mph}$ & SG & Tfe & Paludisme & $\begin{array}{l}\text { Décoction } \\
\text { (or) }\end{array}$ \\
\hline $\begin{array}{l}\text { Salacia } \\
\text { pallescens }\end{array}$ & Sonoufoko & Celastraceae & nph & SG & Tfe & $\begin{array}{c}\text { Retard staturo- } \\
\text { pondéral (enfants) }\end{array}$ & $\begin{array}{l}\text { Décoction } \\
\text { (or) }\end{array}$ \\
\hline $\begin{array}{l}\text { * Sansevieria } \\
\text { liberica }\end{array}$ & Kpayando & Dracaenaceae & $\mathrm{Gr}$ & SG & $\mathrm{Ra}$ & $\begin{array}{c}\text { Paludisme, troubles } \\
\text { hépatiques, fièvre } \\
\text { typhoïde }\end{array}$ & $\begin{array}{l}\text { Décoction } \\
\text { (or) }\end{array}$ \\
\hline $\begin{array}{l}\text { Sarcocephalus } \\
\text { latifolius }\end{array}$ & Kodo & Rubiaceae & $\mathrm{mph}$ & At & $\mathrm{Ra}$ & $\begin{array}{c}\text { Paludisme, ictère, } \\
\text { provoque avortement }\end{array}$ & $\begin{array}{l}\text { Décoction } \\
\text { (or) }\end{array}$ \\
\hline $\begin{array}{l}\text { Schrankia } \\
\text { leptocarpa }\end{array}$ & Ahossiboassa & $\begin{array}{c}\text { Leg.- } \\
\text { Mimosoideae }\end{array}$ & Lnph & $\mathrm{Pt}$ & Tfe & $\begin{array}{l}\text { Accouchement } \\
\text { difficiles }\end{array}$ & $\begin{array}{l}\text { Décoction } \\
\text { (or) }\end{array}$ \\
\hline $\begin{array}{l}\text { Schrebera } \\
\text { arborea }\end{array}$ & Avléni & Oleaceae & $\mathrm{mPh}$ & GC & $\mathrm{Fr}$ & $\begin{array}{l}\text { Fragilité du fontanelle } \\
\text { (nouveau-né) }\end{array}$ & $\begin{array}{l}\text { Décoction } \\
\text { (or) }\end{array}$ \\
\hline
\end{tabular}


A.C. ADOMOU et al. / Int. J. Biol. Chem. Sci. 6(2): 745-772, 2012

\begin{tabular}{|c|c|c|c|c|c|c|c|}
\hline $\begin{array}{l}\text { Schwenckia } \\
\text { americana }\end{array}$ & Zron ou Amankuikui & Solanaceae & $\mathrm{Th}$ & $\mathrm{Pt}$ & Tfe & $\begin{array}{c}\text { Manque de vitalité du } \\
\text { fœtus }\end{array}$ & $\begin{array}{l}\text { Décoction } \\
\text { (or) }\end{array}$ \\
\hline Scoparia dulcis & Vivimantéton & Scrophulariaceae & $\mathrm{Ch}$ & $\mathrm{Pt}$ & Tfe & $\begin{array}{l}\text { Magique (porte } \\
\text { chance) }\end{array}$ & Savon (bain) \\
\hline Secamone afzelii & Zukudjou & Asclepiadaceae & Lmph & $\mathrm{GC}$ & Tfe & $\begin{array}{l}\text { Constipations } \\
\text { (purgatif) }\end{array}$ & $\begin{array}{l}\text { Décoction } \\
\text { (or) }\end{array}$ \\
\hline $\begin{array}{l}\text { Securidaca } \\
\text { longepedunculata }\end{array}$ & Kpatado & Polygalaceae & $\mathrm{mph}$ & At & $\mathrm{Ra}$ & Toux, sinusite & $\begin{array}{l}\text { Décoction } \\
\text { (or) }\end{array}$ \\
\hline * Senna alata & Amanssou & $\begin{array}{c}\text { Leg.- } \\
\text { Caesalpinioideae }\end{array}$ & nph & $\mathrm{Pt}$ & Tfe & Troubles hépatiques & $\begin{array}{l}\text { Décoction } \\
\text { (or) }\end{array}$ \\
\hline * Senna italica & Agbogbé & $\begin{array}{c}\text { Leg.- } \\
\text { Caesalpinioideae }\end{array}$ & $\mathrm{Ch}$ & $\mathrm{Pt}$ & Tfe & $\begin{array}{c}\text { Constipations, selles } \\
\text { difficiles (purgatif) }\end{array}$ & $\begin{array}{l}\text { Décoction } \\
\text { (or) }\end{array}$ \\
\hline Senna obtusifolia & Kpanhu & $\begin{array}{c}\text { Leg.- } \\
\text { Caesalpinioideae }\end{array}$ & $\mathrm{Ch}$ & $\mathrm{Pt}$ & Tfe & Paludisme & $\begin{array}{l}\text { Décoction } \\
\text { (or) }\end{array}$ \\
\hline $\begin{array}{l}\text { Senna } \\
\text { occidentalis }\end{array}$ & Aziman & $\begin{array}{c}\text { Leg.- } \\
\text { Caesalpinioideae }\end{array}$ & $\mathrm{Ch}$ & $\mathrm{Pt}$ & Tfe & Paludisme & $\begin{array}{l}\text { Décoction } \\
\text { (or) }\end{array}$ \\
\hline * Senna siamea & Acacia & $\begin{array}{c}\text { Leg.- } \\
\text { Caesalpinioideae }\end{array}$ & $\mathrm{mPh}$ & $\mathrm{Pt}$ & $\begin{array}{l}\text { Tfe- } \\
\text { Ec, } \\
\mathrm{Ra}\end{array}$ & $\begin{array}{c}\text { Paludisme- } \\
\text { Hypertension, fièvre } \\
\text { typhoïde }\end{array}$ & $\begin{array}{l}\text { Décoction } \\
\text { (or) }\end{array}$ \\
\hline $\begin{array}{l}\text { Sesamum } \\
\text { indicum }\end{array}$ & Agboman & Pedaliaceae & Th & $\mathrm{Pt}$ & Tfe & $\begin{array}{c}\text { Accouchement } \\
\text { difficile }\end{array}$ & $\begin{array}{l}\text { Décoction } \\
\text { (or) }\end{array}$ \\
\hline Sida acuta & Adonman & Malvaceae & $\mathrm{Ch}$ & $\mathrm{Pt}$ & Tfe & $\begin{array}{l}\text { Magique (porte } \\
\text { chance) }\end{array}$ & Savon (bain) \\
\hline $\begin{array}{l}\text { Spathodea } \\
\text { campanulata }\end{array}$ & Adadaman & Bignoniaceae & $\mathrm{mPh}$ & At & Tfe & $\begin{array}{l}\text { Kyste, myome et } \\
\text { fibrome }\end{array}$ & $\begin{array}{l}\text { Décoction } \\
\text { (or) }\end{array}$ \\
\hline $\begin{array}{l}\text { Stereospermum } \\
\text { kunthianum }\end{array}$ & tchékounouhélou & Bignoniaceae & $\mathrm{mph}$ & $\mathrm{SZ}$ & Tfe & $\begin{array}{l}\text { Magique (protection } \\
\text { contre les mauvais }\end{array}$ & Savon (bain) \\
\hline
\end{tabular}


A.C. ADOMOU et al. / Int. J. Biol. Chem. Sci. 6(2): 745-772, 2012

\begin{tabular}{|c|c|c|c|c|c|c|c|}
\hline & & & & & & sorts) & \\
\hline $\begin{array}{l}\text { Strophanthus } \\
\text { hispidus }\end{array}$ & Tchakpa & Apocynaceae & Lmph & $\mathrm{SG}$ & $\mathrm{Ra}$ & Lèpre & $\begin{array}{l}\text { Décoction } \\
\text { (or) }\end{array}$ \\
\hline $\begin{array}{l}\text { Tamarindus } \\
\text { indica }\end{array}$ & Djèvivigoto & $\begin{array}{c}\text { Leg.- } \\
\text { Caesalpinioideae }\end{array}$ & $\mathrm{mPh}$ & $\mathrm{Pt}$ & $\mathrm{Ec}$ & Paludisme & $\begin{array}{l}\text { Décoction } \\
\text { (or) }\end{array}$ \\
\hline Tephrosia villosa & Faux linman & $\begin{array}{c}\text { Leg.- } \\
\text { Papilionoideae }\end{array}$ & $\mathrm{Ch}$ & $\mathrm{Pt}$ & Tfe & Folie & $\begin{array}{l}\text { Décoction } \\
\text { (or) }\end{array}$ \\
\hline $\begin{array}{l}\text { * Tephrosia } \\
\text { vogelii }\end{array}$ & Linman & $\begin{array}{c}\text { Leg.- } \\
\text { Papilionoideae }\end{array}$ & nph & $\mathrm{Pt}$ & Tfe & Folie & $\begin{array}{l}\text { Décoction } \\
\text { (or) }\end{array}$ \\
\hline $\begin{array}{l}\text { Terminalia } \\
\text { glaucescens }\end{array}$ & Alotoudo & Combretaceae & $\mathrm{mPh}$ & $\mathrm{SG}$ & $\mathrm{Ra}$ & Toux & $\begin{array}{l}\text { Décoction } \\
\text { (or) }\end{array}$ \\
\hline $\begin{array}{l}\text { Tetrapleura } \\
\text { tetraptera }\end{array}$ & Linja & Leg.Mimosoideae & $\mathrm{mPh}$ & $\mathrm{GC}$ & Fr & Infections & $\begin{array}{l}\text { Décoction } \\
\text { (or) }\end{array}$ \\
\hline $\begin{array}{l}\text { Trichilia } \\
\text { prieureana }\end{array}$ & Tchivi & Meliaceae & $\mathrm{mph}$ & GC & Tfe & Fièvre & Savon (bain) \\
\hline $\begin{array}{l}\text { Trilepisium } \\
\text { madagascariensis }\end{array}$ & Houndi houndi & Moraceae & $\mathrm{mPh}$ & At & $\mathrm{Ec}$ & Troubles menstruels & $\begin{array}{l}\text { Décoction } \\
\text { (or) }\end{array}$ \\
\hline $\begin{array}{l}\text { * Tylophora } \\
\text { cameroonica }\end{array}$ & Tégbéssou & Asclepiadaceae & Lmph & $\mathrm{GC}$ & Tfe & $\begin{array}{l}\text { Magique (protection } \\
\text { contre les mauvais } \\
\text { sorts) }\end{array}$ & Savon (bain) \\
\hline Uraria picta & Assissihanhu & $\begin{array}{c}\text { Leg.- } \\
\text { Papilionoideae }\end{array}$ & Th & $\mathrm{Pt}$ & Tfe & $\begin{array}{l}\text { Accouchement } \\
\text { difficile }\end{array}$ & $\begin{array}{l}\text { Décoction } \\
\text { (or) }\end{array}$ \\
\hline Uvaria chamae & Ayadahado & Annonaceae & Lmph & $\mathrm{SG}$ & $\mathrm{Ra}$ & Anémie & $\begin{array}{l}\text { Décoction } \\
\text { (or) }\end{array}$ \\
\hline Vernonia cinerea & Hussikonou & Asteraceae & Th & $\mathrm{Pt}$ & Tfe & $\begin{array}{l}\text { Magique (porte } \\
\text { chance) }\end{array}$ & Savon (bain) \\
\hline
\end{tabular}


A.C. ADOMOU et al. / Int. J. Biol. Chem. Sci. 6(2): 745-772, 2012

\begin{tabular}{|c|c|c|c|c|c|c|c|}
\hline $\begin{array}{l}\text { Vitellaria } \\
\text { paradoxa }\end{array}$ & Limigoto & Sapotaceae & $\mathrm{mPh}$ & $S$ & $\mathrm{Ec}$ & $\begin{array}{c}\text { Ulcère, maux de } \\
\text { ventre }\end{array}$ & $\begin{array}{c}\text { Décoction } \\
\text { (or) }\end{array}$ \\
\hline Waltheria indica & Adassoussou & Sterculiaceae & $\mathrm{nph}$ & $\mathrm{Pt}$ & Tfe & $\begin{array}{l}\text { Infections, retard } \\
\text { staturo-pondéral }\end{array}$ & $\begin{array}{l}\text { Décoction } \\
\text { (or) }\end{array}$ \\
\hline $\begin{array}{l}\text { Xylopia } \\
\text { aethiopica }\end{array}$ & Kpédjréku & Annonaceae & $\mathrm{mPh}$ & SG & $\begin{array}{c}\text { Tfe- } \\
\text { Fr }\end{array}$ & Infection & $\begin{array}{c}\text { Décoction } \\
\text { (or) }\end{array}$ \\
\hline $\begin{array}{l}\text { Zanthoxylum } \\
\text { zanthoxyloides }\end{array}$ & Hedo & Rutaceae & mph & SG & $\mathrm{Ra}$ & $\begin{array}{c}\text { Infections (surtout } \\
\text { après accouchements), } \\
\text { caries dentaires }\end{array}$ & $\begin{array}{l}\text { Décoction } \\
\text { (or) }\end{array}$ \\
\hline $\begin{array}{l}\text { * Zingiber } \\
\text { officinale }\end{array}$ & Dotè (blanc et rouge) & Zingiberaceae & Gr & $\mathrm{Pt}$ & $\mathrm{Rh}$ & $\begin{array}{c}\text { Faiblesse sexuelle, } \\
\text { hypertension }\end{array}$ & $\begin{array}{c}\text { Décoction } \\
\text { (or) }\end{array}$ \\
\hline Indéterminée & Fèfègoto & & & & Ec & $\begin{array}{c}\text { Kyste, fibrome, } \\
\text { myome }\end{array}$ & $\begin{array}{l}\text { Décoction } \\
\text { (or) }\end{array}$ \\
\hline \multicolumn{8}{|c|}{ 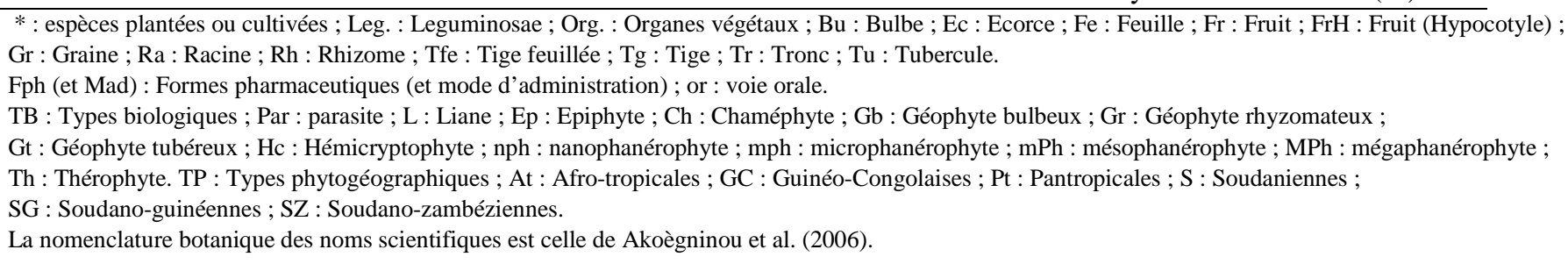 } \\
\hline
\end{tabular}




\section{DISCUSSIONS}

Une enquête de marché conduite par Hermans et al. (2004) au sud du Bénin auprès de 30 vendeuses de plantes médicinales n'a permis de recenser que 85 espèces de plantes antipaludiques. Nouhoum (2010) n'a recensé que 65 espèces végétales suite à l'étude ethnobotanique conduite dans le plus grand marché (marché internationales de Dantokpa) de plantes médicinales au Bénin. L'inventaire de 90 étals de vente de plantes médicinales conduit dans trois marchés au Cameroun a rapporté 30 espèces réparties en 25 familles et 29 genres (Dibong et al., 2011). D'une façon générale, la richesse spécifique par marché suite à l'inventaire des plantes médicinales commercialisées est de l'ordre d'une centaine d'espèces ; les études inventoriant plus de 200 espèces végétales étant rares (Monteiro et al., 2010). Il ressort de la présente étude que la stratégie d'enquête utilisée qui a consisté à focaliser nos investigations sur une seule vendeuse sur la base d'un consensus, a été concluante avec 205 espèces végétales inventoriées. La diversité de plantes médicinales sur un étal de marché pourrait être liée au volume de plantes commercialisées, au degré de spécialisation dans la vente de certaines espèces, à la diversité des provenances des plantes, à l'expérience de la vendeuse. Elle varierait aussi avec les saisons (disponibilité saisonnière des plantes). Il faut aussi souligner la réticence des vendeurs à fournir les informations ethnobotaniques (Monteiro et al., 2010). La présente étude montre que l'estimation de la richesse spécifique des plantes médicinales d'un étal de marché dépend aussi du temps que l'on dispose pour l'inventaire et de la volonté de la vendeuse à coopérer.

Les plantes comme Argemone
mexicana,
Heliotropium indicum, Kalankoe crenata et Chenopodium ambrosioides sont souvent préservées dans les habitations (Akoègninou et al., 2006). Les espèces telles que Piper guineense, Senna italica, Cola acuminata,
Garcinia kola et Picralima nitida sont importées du Nigéria et du Ghana; lesquelles sont rares ou absentes de la flore indigène sauvage du Bénin (Akoègninou et al., 2006).

Les familles botaniques les plus fréquemment recensées sur les étals de marché sont les Leguminosae, Rubiaceae, Euphorbiaceae et Apocynaceae, et sont parmi les plus riches en espèces de plante médicinale (Joy et al., 2001). Une synthèse sur les plantes médicinales commercialisées a montré que les Leguminosae constituent la famille la plus représentée sur les étals de marché (Monteiro et al., 2010).

Le spectre des types biologiques observé concorde avec celui trouvé au Cameroun par Dibong et al. (2011) qui ont recensé $60 \%$ de ligneux contre $40 \%$ d'herbacées. Le spectre biogéographique montre une bonne représentation des espèces à large distribution qui sont des espèces capables de coloniser les milieux dégradés (champs, jachères, bords de routes) sont bien représentées (50\% des espèces recensées). Ces résultats confirment les observations faites par Bussmann et Sharon (2009) au nord de Peru. Cependant, les espèces de forêt humide de la zone guinéo-congolaise (29\%) ne sont pas négligeables et méritent une attention particulière en matière de conservation car la couverture de forêt dense humide est très maigre au Bénin (69557 ha; Anonyme, 2007 ; Anonyme, 2010). Au nombre de ces espèces vulnérables (exploitation des fruits et graines), on peut citer: Trichilia prieureana, Monodora myristica, Schrebera arborea, Tetrapleura tetraptera et Entada gigas.

La prédominance des tiges feuillées (et feuilles), racines, écorces et fruits sur les étals de marché de plantes médicinales est indiquée par plusieurs auteurs (Betti, 2002; Monteiro et al., 2010 ; Dibong et al., 2011). Contrairement aux feuilles et fruits, la disponibilité des écorces et des racines de plantes ligneuses telles que les arbres est très peu influencée par les saisons; elles sont plus disposées à être conservées pendant longtemps (Albuquerque, 2006). Certains organes, surtout les fruits et 
les graines, sont utilisés comme des ingrédients auxiliaires ou adjuvants, permettent de renforcer l'action thérapeutique des composantes principales des recettes et permettent aussi de traiter les symptômes secondaires de la maladie (Joy et al., 2001).

Le spectre des maladies recensées reflète le pattern des maladies courantes au plan national et régional qui montre que le paludisme et les infections demeurent les maladies les plus meurtrières au Bénin et en Afrique tropicale en général (Fourn et al., 2001). En Afrique, les maladies telles que la diarrhée, le paludisme et la pneumonie constituent les causes majeures de la mortalité infantile (Black et al., 2010). Il ressort de la présente étude que les espèces de plantes telles Pterocarpus santalinoides (écorces, feuilles), Psidium guajava (feuilles), Combretum mucronatum (feuilles) et Cnestis ferruginea (feuilles) sont constamment mentionnées pour le traitement de la diarrhée infantile. Les espèces telles que Pavetta crassipes, Pavetta corymbosa, Nauclea diderrichii, Pupalia lappacea, Phyllanthus amarus, Rytigynia umbellulata et Flueggea virosa sont indiquées dans le traitement du paludisme (Hermans et al., 2004).

$\mathrm{Au}$ nombre des espèces dont les organes sont les plus demandés sur le marché et qui se trouvent sur la liste rouge du Bénin (Adomou et al., 2011), on peut citer : Khaya senegalensis, Monodora myristica, Xylopia aethiopica, Tetrapleura tetraptera, Acridocarpus smeathmannii, Cleistopholis patens, Entada gigas, Caesalpinia bonduc, Carissa spinarum etc. Ceci serait dû au fait que la pression anthropique sur ces espèces est très forte. L'exploitation et la commercialisation des organes comme fruit (Xylopia aethiopica), graine (Entada gigas), racine (Acridocarpus smeathmannii) et écorce (Cleistopholis patens) sont préjudiciables pour la survie de l'espèce (Botha et al., 2004).

\section{Conclusion}

Le présent travail a permis de faire une première évaluation de la diversité des plantes médicinales et organes végétaux commercialisés dans la commune d'AbomeyCalavi. La richesse spécifique et la diversité des usages témoignent du niveau de connaissance des plantes médicinales et de la dépendance de la population locale pour les soins de santé primaire. La vendeuse détient un savoir assez étendu sur l'usage des plantes médicinales. L'originalité des données recueillies réside dans le fait que $13 \%$ des espèces d'organes recensés sont qualifiés d'ingrédients auxiliaires par la vendeuse. La stratégie d'enquête utilisée qui a consisté à focaliser nos investigations sur une seule vendeuse sur la base d'un consensus, a été concluante avec 205 espèces végétales inventoriées.

\section{REFERENCES}

Adjanohoun EJ, Adjakidjè V, Ahyi MRA, Aké Assi L, Akoègninou A, d'Almeida J, Apovo F, Boukef K, Chadaré M, Cusset G, Dramane K, Eyme J, Gassita J-N, Gbaguidi N, Goudoté E, Guinko S, Houngnon P, Issa LO, Keita A, Kiniffo HV, Kone-Bamba D, Musampa Nseyya A, Saadou M, Sodogandji TH, de Souza S, Tchabi A, Zinsou Dossa C, Zohoun TH. 1989. Contribution aux Etudes Ethnobotaniques et Floristiques en République Populaire du Bénin. Agence de Coopération Culturelle et Techniques : Paris ; $895 \mathrm{p}$.

Adjanohoun EJ, Eyme J, Dramane KL, Fouraste L, Keita LE, Bras M, Lejoly J, Penge O \& Waechter P. 1996. Revue de Médicine et Pharmacopées Africaines (vol. 10). Editions GRIPT: $138 \mathrm{p}$

Adomou AC, Agbani OP, Sinsin B. 2011. Plantes. In Protection de la Nature en Afrique de l'Ouest: Une Liste Rouge pour le Bénin. Nature Conservation in West Africa: Red List for Benin, Neuenschwander P, Sinsin B, Goergen G (eds). International Institute of Tropical Agriculture: Ibadan, Nigeria; 21-46.

Adomou AC, Sinsin B, Akoègninou A, Van der Maesen J. 2009. Plant species and 
ecosystems with high conservation priority in Benin. In Systematics and Conservation of African Plants, Van der Burgt X, van der Maesen J, Onana JM (eds). Royal Botanic Gardens: Kew; 427441.

Aké Assi L. 2001. Flore de la Côte-d'Ivoire: catalogue systématique, biogéographie et écologie I. Boissiera, 57: 1-396.

Aké Assi L. 2002. Flore de la Côte-d'Ivoire: catalogue systématique, biogéographie et écologie II. Boissiera, 58: 1-401.

Akoègninou A. 2004. Recherches botaniques et écologiques sur les forêts actuelles du Bénin. Thèse d'Etat, Université de Cocody-Abidjan, 326p.

Akoègninou $\mathrm{A}$, van der Burg $\mathrm{WJ}$, van der Maesen LJG. 2006. Flore Analytique $d u$ Bénin. Backhuys Publishers: Wageningen; p.1034.

Albuquerque UP. 2006. Re-examining hypothesis concerning the use and knowledge of medicinal plants: A study in Caatinga vegetation of NE Brazil. Journal of Ethnobiology and Ethnomedicine, 2: 30-36.

Albuquerque UP, Monteiro JM, Ramos MA, Amorim ELC. 2007. Medicinal and magic plants from a public market in northeastern Brazil. Journal of Ethnopharmacology, 110: 76-91.

Betti JL. 2002. Medicinal plants sold in Yaoundé markets, Cameroon. African Study Monographs, 23(2): 47-64.

Black RE, Cousens S, Johnson HL, Lawn JE, Rudan I, Bassani DG, Jha P, Campbell H, Walker CF, Cibulskis R, Eisele T, Liu L, Mathers C. 2010. Global, regional, and national causes of child mortality in 2008: a systematic analysis. The Lancet, $\mathbf{3 7 5}$ (9730): 1969-1987.

Botha J, Witkowski ETF, Shackleton CMA. 2004. Market profiles and trade in medicinal plants in the Lowveld, South Africa. Environmental Conservation, 31(1): 38-46.

Bussmann RW, Sharon D. 2009. Markets, Healers, Vendors, Collectors: The
Sustainability of Medicinal Plant Use in Northern Peru. Mountain Research and Development, 29(2): 128-134.

Cunningham AB. 1993. African medicinal plants. Setting priorities at the interface between conservation and primary healthcare. People and Plants Working Paper, (vol 1). UNESCO: Paris; 50.

Dibong SD, Mpondo Mpondo E, Ngoye A, Kwin MF, Betti JL. 2011. Ethnobotanique et phytomédecine des plantes médicinales de Douala, Cameroun. Journal of Applied Biosciences, 37: 2496 - 2507.

FAO 2010. Evaluation des ressources forestières mondiales. Bénin, Rapport national. Départ. des Forêts, ONU/ Rhome.

Fourn L, Sakou G, Zohoun T. 2001. Utilisation des services de santé par les mères des enfants fébriles au sud du Bénin. Santé Publique, 13: 161-168.

Gil R, Mejías R, Carmona J, Mejías R, Arredondo MR. 2003. Estúdio etnobotánico de algunas plantas medicinales expendidas em los herbolarios de Mérida, Ejido y Tabay (Estado Mérida-Venezuela). Revista de la Facultad de Farmácia, 45(1): 69-46.

Hermans M, Akoègninou A, van der Maesen LJG. 2004. Medicinal plants used to treat malaria in southern Benin. Economic Botany, 58: S239-S252.

IFN. 2007. Inventaire Forestier National. MEPN/DFRN/DFS/CENATEL, Cotonou, Bénin.

INSAE-RGPH. 2002. Recensement général de la population et de l'habitat. INSAE, Cotonou, Bénin.

Jiofack T, Fokunang C, Guedje N, Kemeuze $\mathrm{V}$, Fongnzossie E, Nkongmeneck BA, Mapongmetsem PM, Tsabang N. 2010. Ethnobotanical uses of medicinals plants of two ethnoecological regions of Cameroon. International Journal of Medicine and Medical Sciences, 2(3): 6079. 
Joy PP, Thomas J, Mathew S, Skaria BP. 2001. Medicinal Plants. Tropical Horticulture, 2: 449-632.

Maiga A, Diallo D, Fane S, Sanogo R, Paulsen BS, Cisse B. 2005. A survey of toxic plants on the market in the district of Bamako, Mali: traditional knowledge compared with a literature search of modern pharmacology and toxicology. Journal of Ethnopharmacology, 96: 183193.

Monteiro JM, Araujo LE, Amorim ELC, Albuquerque UP. 2010. Local markets and medicinal plant commerce: a review with emphasis on Brazil. Economic Botany, 64(4): 352-366.

Nouhoum S. 2010. Etude ethnobotanique des plantes médicinales vendues au marchéDantokpa de Cotonou (Bénin). Mémoire de Licence, EPAC, Univ. d'Abomey-Calavi, Bénin.
Olsen CS. 2005. Trade and conservation of Himalayan medicinal plants: Nardostachys grandiflora DC and Neopicrorhiza scrophulariiflora (Pennell) Hong. Biol. Conserv., 125: 505-514.

PAMPLONA G. 1999. Guide des Plantes Médicinales (Vols 1 et 2). Editions Vie et Santé; 736p.

Pei S. 2001. Ethnobotanical Approaches of traditional medicine studies: some experiences from Asia. Pharm. Bot., 39: 74-79.

Raunkiaer C. 1934.The Life Forms of Plants and Statistical Plant Geography. Clarendron Press: Oxford; 632 p.

White F. 1983. The vegetation of Africa, a descriptive memoir to accompany the UNESCO / AETFAT / UNSO /UNESCO. Natural Resources Research, 20: 1-356. 